\title{
Application of the locality principle to radio occultation studies of the Earth's atmosphere and ionosphere
}

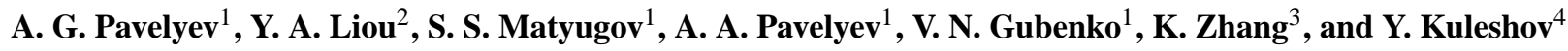 \\ ${ }^{1}$ Kotelnikov Institute of Radio Engineering and Electronics of the Russian Academy of Sciences, Fryazino, Moscow region, \\ Russia \\ ${ }^{2}$ Center for Space and Remote Sensing Research, National Central University, Jhong-Li, 320, Taiwan \\ ${ }^{3}$ SPACE Research Centre/RMIT University/Australia (03) 99253272, Melbourne, Australia \\ ${ }^{4}$ National Climate Centre, Bureau of Meteorology, Melbourne, Australia \\ Correspondence to: A. G. Pavelyev (alxndr38@mail.ru) and Y. A. Liou (yueian@csrsr.ncu.edu.tw)
}

Received: 23 October 2014 - Published in Atmos. Meas. Tech. Discuss.: 20 January 2015

Revised: 8 June 2015 - Accepted: 22 June 2015 - Published: 17 July 2015

\begin{abstract}
A new formulation of the previously introduced principle of locality is presented. The principle can be applied for modernization of the radio occultation (RO) remote sensing of the atmospheres and ionospheres of the Earth and other planets. The principle states that significant contributions to variations of the intensity and phase of the radio waves passing through a layered medium are connected with influence of the vicinities of tangential points where the refractivity gradient is perpendicular to the radio ray trajectory. The RO method assumes spherical symmetry of the investigated medium. In this case, if location of a tangent point relative to the spherical symmetry centre is known, the time derivatives of the RO signal phase and Doppler frequency variations can be recalculated into the refractive attenuation. Several important findings are consequences of the locality principle: (i) if position of the centre of symmetry is known, the total absorption along the ray path can be determined at a single frequency; (ii) in the case of low absorption the height, displacement from the radio ray perigee, and tilt of the inclined ionospheric (atmospheric) layers can be evaluated; (iii) the contributions of the layered and irregular structures in the RO signal can be separated and parameters of layers and turbulence can be measured at a single frequency using joint analysis of the intensity and phase variations. Specially for the Earth's troposphere, the altitude distributions of the weak total absorption (about of $1-4 \mathrm{db}$ ) of the radio waves at GPS frequencies corresponding to possible influence of the oxygen, water vapour, and hydrometeors can be measured with accuracy of about $0.1 \mathrm{db}$ at a single frequency. In accor-
\end{abstract}

dance with the locality principle, a new index of ionospheric activity is introduced. This index is measured from the phase variations of radio waves passing through the ionosphere. Its high correlation with the $\mathrm{S} 4$ scintillation index is established. This correlation indicates the significant influence of locally spherical symmetric ionospheric layers on variations of the phase and intensity of the RO signal passing through transionospheric communication links. Obtained results expand applicable domain of the RO method as a powerful remote sensing technique for geophysical and meteorological research.

\section{Introduction}

The radio occultation (RO) remote sensing has been known for the last 50 years as a powerful tool for investigation of the atmospheres, ionospheres and planetary surfaces (Fjeldbo, 1964; Marouf and Tyler, 1986; Lindal et al., 1983, 1987; Hinson et al., 1997, 1999; Yunck et al., 2000; Yakovlev, 2002, and references therein). With regard to the study of near-Earth space, the RO method should be competitive with other means of remote sensing (Gurvich and Krasilnikova, 1987; Yunck et al., 1988; Melbourne et al., 1994; Steiner et al., 1999; Beyerle and Hocke, 2001; Beyerle et al., 2001, 2002; Yakovlev, 2002; Liou et al., 2002, 2003, 2005a, 2010; Manzini and Bengtsson, 2008; Anthes, 2011). Assumption of spherical symmetry - cornerstone of RO method - should be carefully analyzed when the RO technology is applied to 
global monitoring of the Earth's ionosphere and atmosphere at different altitudes (Vorob'ev and Krasilnikova, 1994; Melbourne et al., 1994; Syndergaard, 1998, 1999; Yunck et al., 2000). In particular, effectiveness of the RO method applied for investigation of the Earth's ionosphere can be compared with radio tomographic approach (Kunitsyn and Tereshchenko, 2003). The tomographic method allows obtaining 2-D distributions of electron density in the ionosphere using a chain of ground-based receivers, which capture signals of low Earth orbital (LEO) or navigational satellites along a set of intersecting radio rays (Kunitsyn et al., 2011, 2013). Unlike the radio tomographic approach, the RO method uses a set of nearly parallel radio-ray trajectories. The RO method applied for processing the assumption of spherical symmetry of the Earth's ionosphere and atmosphere with known location of the centre of symmetry (Melbourne et al., 1994; Yakovlev, 2002; Melbourne, 2004). In accordance with this assumption, all resulting altitude profiles of atmospheric and ionospheric parameters are attached to vertical and horizontal coordinates of the radio ray perigee relative to a spherical symmetry centre, which is close to or coincident with the centre of the Earth or a selected planet.

Highly stable signals synchronized by atomic frequency standards and radiated by GPS satellites at frequencies $\mathrm{F} 1=1575.42 \mathrm{MHz}$ and $\mathrm{F} 2=1227.60 \mathrm{MHz}$ create at the altitudes from 0 to $20000 \mathrm{~km}$ radio fields that can be used for the development of the RO method as a new tool for global monitoring of the ionosphere and neutral atmosphere (Gurvich and Krasilnikova, 1987; Yunck, 1988). Several LEO missions were launched during 1995-2014 for the study of the atmosphere and ionosphere: GPS/MET (Melbourne et al., 1994; Ware et al., 1996; Gorbunov et al., 1996; Kursinski et al., 1997; Vorob'ev et al., 1997; Hajj and Romans, 1998), SAC-C (Schmidt et al., 2005), CHAMP (Wickert et al., 2001, 2009), FORMOSAT-3 (Liou et al., 2007; Fong et al., 2008), GRACE (Hajj et al., 2004; Wickert et al., 2005), METOP (Von Engeln et al., 2011; Joo et al., 2012), TERRA-SAR, TANDEM-X (Zus et al., 2014), and FY-3 CNOS (Bai et al., 2014). The success of these missions demonstrated that the $\mathrm{RO}$ technique is a powerful remote sensing tool for obtaining key vertical profiles of bending angle, refractivity, temperature, pressure and water vapour in the atmosphere and electron density in ionosphere with global coverage, high spatial and temporal resolution (Zhang et al., 2013). Explicit analysis of experimental data of LEO missions introduced important contributions in the following areas: (i) the theory of radio wave propagation (Gorbunov and Gurvich, 1998a; Gorbunov et al., 2002; Benzon et al., 2003; Gorbunov and Lauritsen, 2004; Gorbunov and Kirchengast, 2005; Pavelyev et al., 2004, 2010a); (ii) climate changes detection (Kirchengast et al., 2000; Steiner et al., 2001; Foelsche et al., 2008); (iii) space weather effects and ionosphere monitoring (Rius et al., 1998; Jakowski et al., 2004; Wickert et al., 2004; Arras et al., 2008, 2010); (iv) deriving new radio-holographic methods of the RO remote sensing (Karayel and Hinson,
1997; Mortensen and Hoeg, 1998; Pavelyev, 1998; Gorbunov and Gurvich, 1998b; Mortensen et al., 1999; Hocke et al., 1999; Sokolovskiy, 2000; Sokolovskiy et al., 2002; Gorbunov, 2002a, b; Gorbunov et al., 1996, 2002, 2010; Igarashi et al., 2000; Jensen et al., 2003, 2004; Pavelyev et al., 2002, 2004, 2010a, b, 2012; Liou et al., 2010; Yakovlev et al., 2010).

Recently, an important connection between the intensity and time derivatives of the phase, eikonal, Doppler frequency of radio waves propagating through the ionosphere and atmosphere has been discovered by theoretical analysis and confirmed by processing of the RO radio-holograms (Liou and Pavelyev, 2006; Liou et al., 2007, 2010; Pavelyev et al., 2008a, 2012). This connection is a key regularity of the RO method. Now this relationship gets a possibility to recognize that the phase (eikonal) acceleration (proportional to the time derivative of the Doppler shift) has the same importance for the theory of radio waves propagation in a layered medium and solution of the RO inverse problem as the Doppler frequency, phase path excess, and refractive attenuation of the RO signal (Liou and Pavelyev, 2006; Liou et al., 2007; Pavelyev, 2008, 2013; Pavelyev et al., 2009, 2010a, b, $2012,2013)$. It follows from this connection that the phase acceleration technique allows one to convert the phase and Doppler frequency changes into refractive attenuation variations at a single frequency. Note that this is similar to classical dynamics when the derivations of the path and velocity on time and acceleration are connected by the Newton's laws. From such derived refractive attenuation and intensity data, one can estimate the integral absorption of radio waves. This is important for future RO missions for measuring water vapour and minor atmospheric gas constituents, because the difficulty of removing the refractive attenuation effect from the intensity data can be avoided. The phase acceleration/intensity technique can be applied also for determining the location and inclination of sharp layered plasma structures (including sporadic Es layers) in the ionosphere. Advantages of the phase acceleration/intensity technique are validated by analyzing the RO data from the Challenging Minisatellite Payload (CHAMP) and the FORMOSA Satellite Constellation Observing Systems for Meteorology, Ionosphere, and Climate missions (FORMOSAT-3/COSMIC).

The locality principle generalizes the phase path excess acceleration/intensity technique to the practically important case in which the location of the symmetry centre of layered medium is unknown (Pavelyev, 2013; Pavelyev et al., 2012). New relationships have been revealed to expand the scope and applicable domain of the RO method. These relationships allow, in particular, measuring the real height, inclination, and displacement of atmospheric and ionospheric layers from the RO ray perigee relative to the Earth's (or other planetary) surface. This implies the possibility of determining the position and orientation of the fronts of internal waves, which opens a new RO area in geophysical applications for remote sensing of the internal waves in the atmospheres and iono- 
spheres of Earth and other planets (Steiner and Kirchengast, 2000; Liou et al., 2003, 2005b, 2007; Pavelyev et al., 2007; Gubenko et al., 2008a, b, 2011).

The goals of this paper are the following: (i) to formulate a principle of locality; (ii) to present several important findings arising from the locality principle; and (iii) to introduce a new index of ionospheric activity. The paper is structured as follows. In Sect. 2 the formulation of the locality principle is presented. Section 3 describes three important findings following from the locality principle: (i) a possibility to determine the total absorption at a single frequency; (ii) a possibility to evaluate the height, displacement from the radio ray perigee, and tilt of the inclined ionospheric (atmospheric) layers; (iii) method for separation of the contributions of the layered and irregular structures in the RO signal, and technique for measurement of parameters of layers and turbulence at a single frequency using joint analysis of the amplitude and phase variations. In Sect. 4 a new scintillation index based on the refractive attenuation found from the phase variations of the RO signal is introduced and its correlation with the $\mathrm{S} 4$ index is established. Conclusions are given in Sect. 5.

\section{Principle of locality}

The principle of locality is based on a previously established connection (Liou and Pavelyev, 2006; Liou et al., 2007; Pavelyev et al., 2008a, b, 2009, 2010a), which relates the eikonal acceleration $a$ and refractive attenuation $X_{\mathrm{p}}^{\prime}(t)$ of the RO signal emitted by a transmitter $G$ and received by satellite $L$ after passing through a spherically symmetric medium with a centre of symmetry at point $O^{\prime}$ (Fig. 1):

$$
\begin{aligned}
1- & X_{\mathrm{p}}^{\prime}(t)=m^{\prime} a, a=\frac{d^{2} \Phi(t)}{\mathrm{d} t^{2}}=\lambda \frac{\mathrm{d} F_{\mathrm{d}}(t)}{\mathrm{d} t}, m^{\prime} \\
& =\frac{d_{2}^{\prime} d_{1}^{\prime}}{\left(d_{1}^{\prime}+d_{2}^{\prime}\right)}\left(\mathrm{d} p_{\mathrm{s}}^{\prime} / \mathrm{d} t\right)^{-2} ; p_{\mathrm{s}}^{\prime}=\left|O^{\prime} D^{\prime}\right| ; \Phi(t) \\
& =\int_{\mathrm{G}}^{\mathrm{L}} n(l) \mathrm{d} l-R_{0},
\end{aligned}
$$

where $\lambda$ is the length of radio waves; $d_{1}^{\prime}, d_{2}^{\prime}$, and $R_{0}$ are the distances along the straight lines $G D^{\prime}, D^{\prime} L$ and $G L$, respectively; point $D^{\prime}$ is the projection of the centre of symmetry $O^{\prime}$ onto the straight line $G D L$; point $D$ is the projection of the centre $O$ on the straight line $G L ; p_{\mathrm{s}}^{\prime}$ is the impact parameter of the straight line $G D L$ relative to the centre $O^{\prime} ; \Phi(t)$ is the difference between the eikonal of radio waves propagating along the radio ray trajectory $G T L$ and the length $G D L$ as a function of time $t ; n(l)$ is the refractive index; $\mathrm{d} l$ is the differential length of the radio ray $G T L$; and point $T$ is the radio ray perigee having the altitude $h$ relative to the Earth's surface (or that of another planet) (Fig. 1). Another important geometric parameter is the height $H$ of the line of

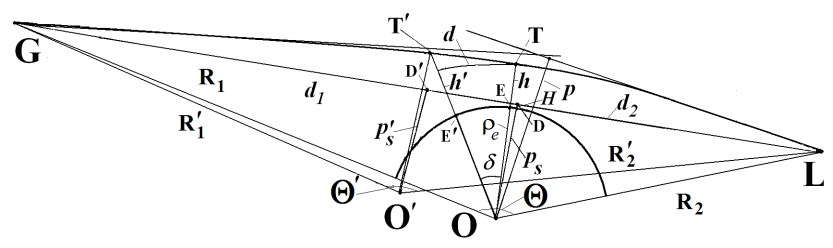

Figure 1. Scheme of radio occultation measurements.

sight $G D L$ above the surface. During a RO event the magnitude $H$ changes from positive to negative values. The eikonal acceleration $a$ (Eq. 1) is proportional to the time derivative of the Doppler frequency of radio waves $F_{\mathrm{d}}(t)$. Equality (Eq. 1) is fulfilled under the following conditions (Liou et al., 2007; Pavelyev et al., 2008a, b):

$$
\mid \begin{aligned}
& \left(p^{\prime}-p_{\mathrm{s}}^{\prime}\right) \frac{\mathrm{d} R_{1,2}^{\prime}}{\mathrm{d} t}|\ll| p_{\mathrm{s}}^{\prime} \frac{\mathrm{d} p_{\mathrm{s}}^{\prime}}{\mathrm{d} t} \mid ; d_{1}^{\prime} \gg d_{2}^{\prime} ; \\
& \left(p^{\prime}-p_{\mathrm{s}}^{\prime}\right) \frac{d}{\mathrm{~d} t}\left(\frac{\partial \theta^{\prime}}{\partial p_{\mathrm{s}}^{\prime}} \frac{\mathrm{d} p_{\mathrm{s}}^{\prime}}{\mathrm{d} t}\right)|\ll|\left(\frac{\mathrm{d} p^{\prime}}{\mathrm{d} t}-\frac{\mathrm{d} p_{\mathrm{s}}^{\prime}}{\mathrm{d} t}\right) \frac{\partial \theta^{\prime}}{\partial p_{\mathrm{s}}^{\prime}} \frac{\mathrm{d} p_{\mathrm{s}}^{\prime}}{\mathrm{d} t} \mid .
\end{aligned}
$$

where $p^{\prime}$ is the impact parameter of the ray $G T L$ relative to the centre $O^{\prime}$. In the case of the RO ionospheric research the centre of spherical symmetry can be shifted relative to the centre $O$, and the first and third inequalities are satisfied only if the distance $O O^{\prime}$ is significantly less than the Earth's radius $\rho_{\mathrm{e}}$ (Fig. 1). The second inequality is necessary for excluding uncertainty because of symmetry in the dependence of the coefficient $m^{\prime}$ with respect to variables $d_{2}^{\prime}, d_{1}^{\prime}$. In the case of GPS RO atmospheric sounding, these inequalities are satisfied if the orbits of satellites $G$ and $L$ are circular, the centre of spherical symmetry $O^{\prime}$ is almost identical to the centre of the Earth (or another planet) $O$, and point $T^{\prime}$ coincides with perigee $T$ of the ray $G T L$ (Fig. 1). Location of the radio ray perigee $T$ in accordance with solution of the RO inverse problem determines temporal dependencies of the height $h$ above the Earth's surface and horizontal coordinates of atmospheric layers (Melbourne et al, 1994; Yakovlev, 2002; Melbourne, 2004).

When absorption is absent, the refractive attenuation $X_{\mathrm{p}}^{\prime}(t)$ found from (Eq. 1) should be equal to the refractive attenuation $X_{\mathrm{a}}(t)$ determined using the RO intensity data (Liou and Pavelyev, 2006):

$X_{\mathrm{p}}^{\prime}(t) \equiv X_{\mathrm{a}}(t) ; X_{\mathrm{a}}(t)=I / I_{0}$,

where $I_{0}$ and $I$ are intensities of the RO signal before and after the moment when radio ray enters the medium, respectively. Identity (Eq. 3) is fulfilled if the coefficient $m^{\prime}$ in Eq. (1) is evaluated in accordance with location of the tangential point $T^{\prime}$, which is the perigee of radio ray $G T L$ relative to the centre of symmetry $O^{\prime}$ (Pavelyev et al., 2013). The refractive attenuation $X_{\mathrm{a}}(t)$ measured from the RO signal intensity data does not depend on position of the 

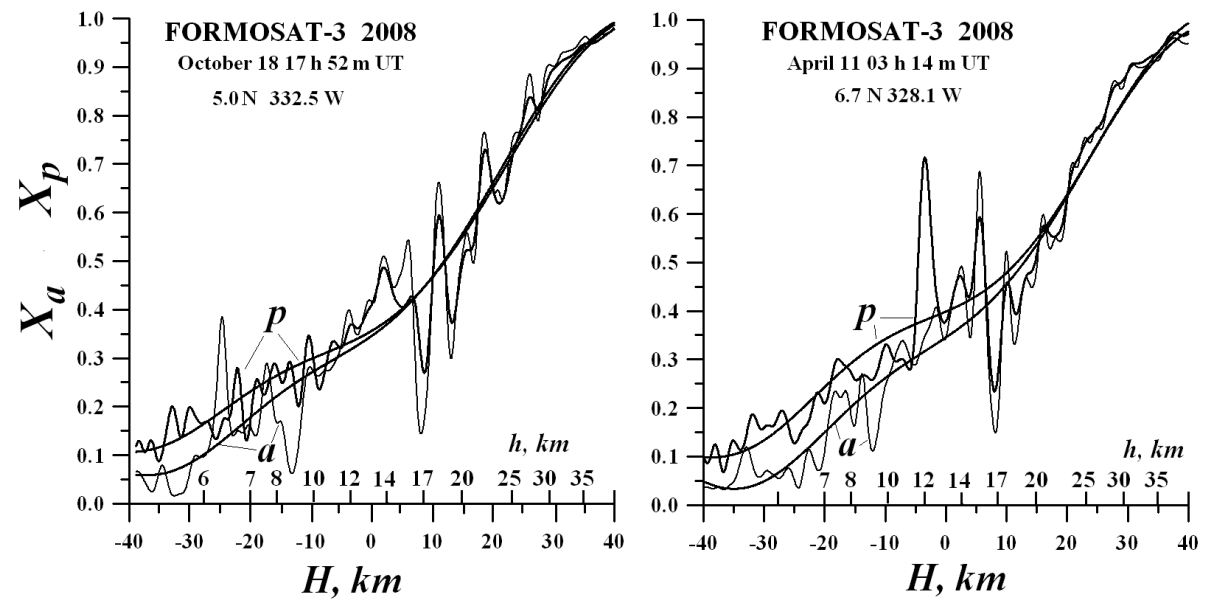

Figure 2. Comparison of the refractive attenuations $X_{\mathrm{a}}(h)$ and $X_{\mathrm{p}}(h)$ and their polynomial approximations, corresponding to the FORMOSAT-3 RO measurements carried out on 18 October and 11 April 2008 (left and right plots, respectively). Thick and thin rough curves (marked by indices "p" and "a") describe the vertical profiles of $X_{\mathrm{p}}(h)$ and $X_{\mathrm{a}}(h)$, respectively. Smooth curves describing polynomial approximations of the altitudes dependences of $X_{\mathrm{p}}(h)$ and $X_{\mathrm{a}}(h)$ are also highlighted by indices "p" and "a".

point $T^{\prime}$ on the ray $G T L$ and, naturally, on coefficient $m^{\prime}$. However, calculated value of the refractive attenuation $X_{\mathrm{p}}^{\prime}(t)$ does depend on the coefficient $m^{\prime}$ (Eq. 1) and location of the tangent point $T^{\prime}$ on the ray $G T L$. This permits to formulate principle of locality under the conditions of single-ray radio wave propagation and absence of absorption (Pavelyev et al., 2012, 2013; Pavelyev, 2013): the refractive attenuations $X_{\mathrm{p}}^{\prime}(t)$ and $X_{\mathrm{a}}(t)$ are equal if evaluation of $X_{\mathrm{p}}^{\prime}(t)$ is provided with the coefficient $m^{\prime}$ corresponding to locations of the spherical symmetry centre $O^{\prime}$ and ray perigee $T^{\prime}$. In accordance with the locality principle, the rapid amplitude and phase variations of the radio waves registered at point $L$ may be considered as connected with influence of a small neighbourhood of the ray perigee $T^{\prime}$ corresponding to the local spherical symmetry centre $O^{\prime}$.

\section{Consequences of the locality principle}

Next important findings that follow the locality principle are addressed below.

\subsection{Possibility of determination of the total absorption}

If location of the symmetry centre is known (for example, when the point $O^{\prime}$ coincides with the Earth's centre $O$ ), the total absorption $\Gamma$ in the atmosphere (ionosphere) can be defined by eliminating from value $X_{\mathrm{a}}(t)$ the refractive attenuation $X_{\mathrm{p}}(h)$ found from eikonal variations.

$\Gamma=10 \lg \frac{X_{\mathrm{a}}(t)}{X_{\mathrm{p}}(t)}$

Some results of determination of the refractive attenuations $X_{\mathrm{a}}(h)$ and $X_{\mathrm{p}}(h)$, and total absorption $\Gamma$ are considered below. Figure 2 (left and right plots) shows two vertical profiles of the refractive attenuations $X_{\mathrm{a}}(h)$ and $X_{\mathrm{a}}(h)$ highlighted by indices "a" and "p" (rough curves), respectively, and their polynomial approximation (smooth curves) measured at the first GPS frequency F1. The measurements were provided in 2008 above two regions located in central Africa (RO events 18 October 17:52 UT (left), and 11 April 03:14 UT (right), with geographical coordinates $5.0^{\circ} \mathrm{N} 332.5^{\circ} \mathrm{W}$, and $6.7^{\circ} \mathrm{N}$ $328.1^{\circ} \mathrm{W}$, respectively) using the FORMOSAT-3 satellites. The altitude dependences of $X_{\mathrm{a}}(h)$ and $X_{\mathrm{p}}(h)$ and their polynomial approximations are described by corresponding pairs of rough and smooth curves indicated by indices "a" and "p", respectively. Values of the altitudes of the line of sight $G D L H$ and the height of the ray perigee $h$ are plotted on the horizontal axis. The $X_{\mathrm{a}}(h)$ and $X_{\mathrm{p}}(h)$ profiles and their polynomial approximations are almost coincident at the heights between 12 and $40 \mathrm{~km}$, and significantly different below 8-9 km (Fig. 2, right and left plots). The correlation between variations of $X_{\mathrm{a}}(h)$ and $X_{\mathrm{p}}(h)$ gradually decreases with height $H$ and magnitude of $X_{\mathrm{a}}(h)$ is obviously well below the corresponding values of $X_{\mathrm{p}}(h)$ in the $5-9 \mathrm{~km}$ altitude interval $h$. This indicates a possible influence of total absorption of the radio waves in the atmosphere.

Other results obtained from the RO experiments carried out during four events on 5 June 2008, are shown in Fig. 3 (left part, groups of curves I-IY) and correspond to measurements of the refractive attenuations $X_{\mathrm{p}}(h)$ and $X_{\mathrm{a}}(h)$ at GPS frequency F1. These RO experiments were conducted in the following areas: Norwegian Sea (I), the eastern Siberian Plateau (II), and Alaska (III, IY). The time and geographical coordinates of these RO events are the following: $16: 18 \mathrm{UT} ; 70.8^{\circ} \mathrm{N} 1.7^{\circ} \mathrm{W}$ (curves I); $13: 28 \mathrm{UT} ; 60.6^{\circ} \mathrm{N}$ $252.5^{\circ} \mathrm{W}$ (curves II); 02:42 UT; $64.9^{\circ} \mathrm{N} 139.7^{\circ} \mathrm{W}$ (curves III); and 10:44 UT; $60.0^{\circ} \mathrm{N} 155.8^{\circ} \mathrm{W}$ (curves IY). A weak but perceptible absorption in 1-2 db interval at frequency F1 

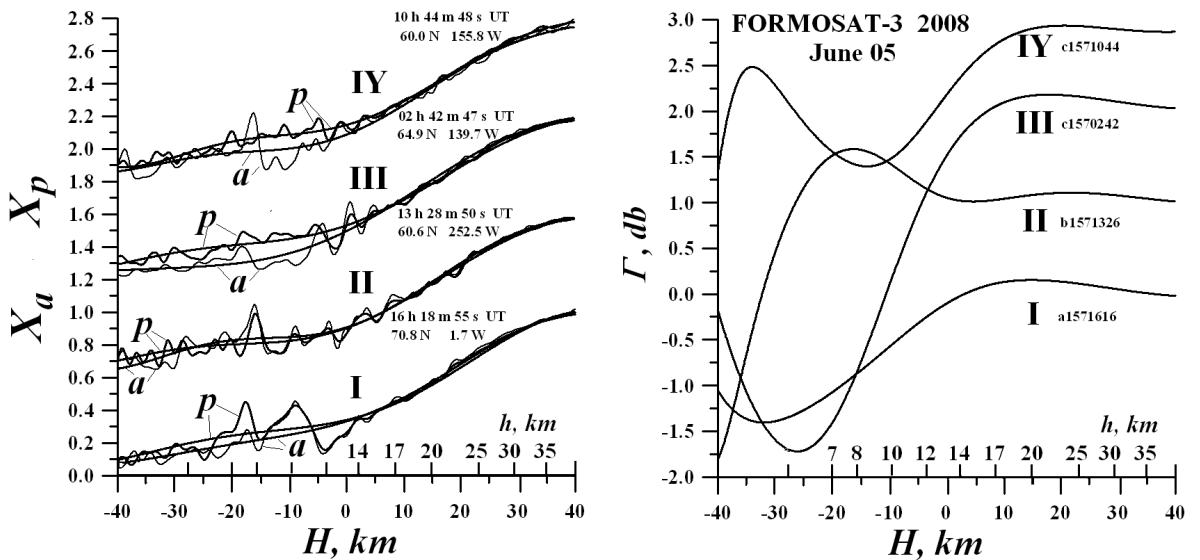

Figure 3. Left plot - Comparison of the refractive attenuations $X_{\mathrm{a}}(h)$ and $X_{\mathrm{p}}(h)$ (groups of curves I-IY). Each group consists of four curves. In each group thick and thin rough curves (marked by indices "p" and "a") describe the experimental vertical profiles of $X_{\mathrm{p}}(h)$ and $X_{\mathrm{a}}(h)$, respectively. Smooth curves in each group describe polynomial approximations of the altitudes dependences of $X_{\mathrm{p}}(h)$ and $X_{\mathrm{a}}(h)$ and are also highlighted by indices " $p$ " and "a", respectively. For convenience groups of curves II-IY are displaced by $0.6 ; 1.2 ; 1.8$ units. Right - The total absorption $\Gamma$ corresponding to the refractive attenuations $X_{\mathrm{a}}(h), X_{\mathrm{p}}(h)$ measured at frequency F1 has been calculated from the smooth curves I-IY (marked by indices $a, p$ in left panel). Curves II, III, and IY are shifted for comparison by 1,2 , and $3 \mathrm{db}$, respectively.
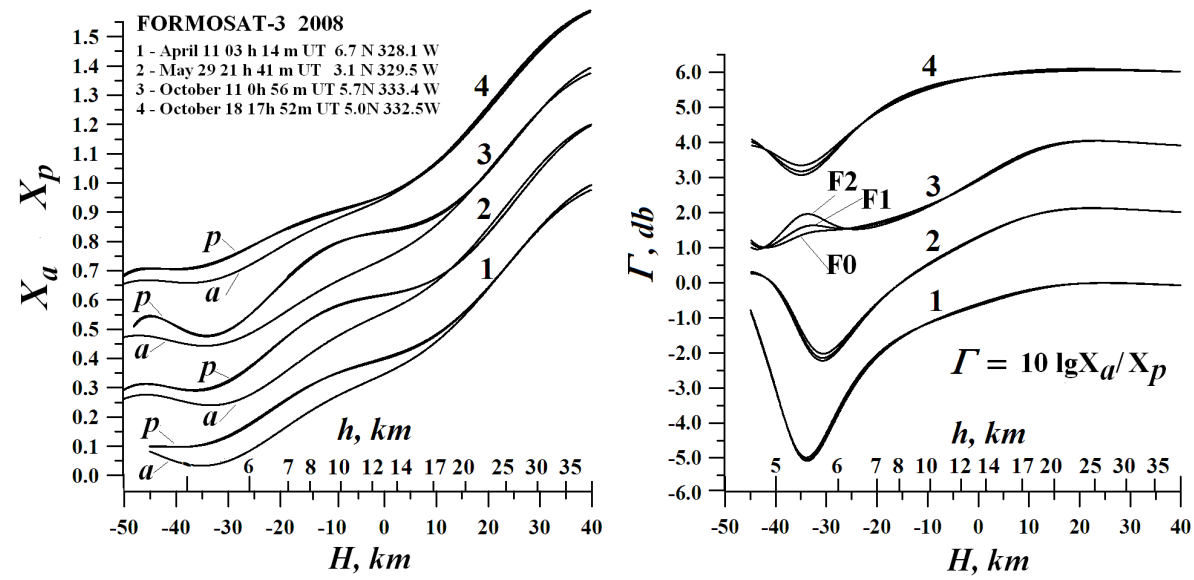

Figure 4. Left plot - Comparison of the polynomial approximations of refractive attenuations $X_{\mathrm{a}}(h), X_{\mathrm{p}}(h)$ (curves $1-4$, indexes "p" and "a", respectively). Curves $2-4$ are displaced for convenience by $0.6 ; 0.4 ; 0.2$, respectively. Right - The total absorption $\Gamma$ corresponding to the refractive attenuations $X_{\mathrm{a}}(h), X_{\mathrm{p}}(h)$ has been calculated from the curves 1-4 (left panel). Curves 2, 3, and 4 are shifted for convenience by 2,4 , and $6 \mathrm{db}$, respectively.

is observed below $8 \mathrm{~km}$ altitude. However, the altitude dependence of absorption $\Gamma$ is different in the considered regions. Two cases revealed significant decrease of absorption $\Gamma$ in the 5-6 km (IY) and 7-8 km (II) height interval. In the remaining areas the total absorption changes from $1.5 \mathrm{db}(\mathrm{I})$ up to $3.5 \mathrm{db}$ (III) below $7 \mathrm{~km}$ altitude $h$.

Polynomial approximations of the refractive attenuations $X_{\mathrm{a}}(h)$ (measured at frequency $\left.\mathrm{F} 1\right) ; X_{\mathrm{p} 1}(h), X_{\mathrm{p} 2}(h)$ (evaluated from the eikonal data at frequencies F1, F2); and $X_{\mathrm{p} 0}(h)$ (estimated from the combined eikonal $F_{0}=\left(F_{1}^{2} \Phi_{1}-\right.$ $\left.\left.F_{2}^{2} \Phi_{2}\right) /\left(F_{1}^{2}-F_{2}^{2}\right)\right)$ are shown in Fig. 4 . These results are relevant to four RO events and correspond to four equatorial regions located in central Africa. Figure 4 (left plot) shows vertical profiles of refractive attenuations $X_{\mathrm{a}}(h), X_{\mathrm{p} 1}(h)$ found from the FORMOSAT-3 RO data corresponding to four events carried out on 11 April 2008 03:14 UT, 6.7 $7^{\circ} \mathrm{N}$ $328.1^{\circ} \mathrm{W} ; 29$ May $21: 41 \mathrm{UT} 3.1^{\circ} \mathrm{N} 329.5^{\circ} \mathrm{W} ; 11$ October 00:56 UT, $5.7^{\circ} \mathrm{N} 333.4^{\circ} \mathrm{W}$; and 18 October 17:52 UT, $5.0^{\circ} \mathrm{N}$ $332.5^{\circ} \mathrm{W}$; (curves $1-4$, respectively). Values of the altitudes $h$ of the ray perigee $T$ and the height $H$ of the straight line $G D L$ relative to the Earth's surface are marked on the horizontal axis. The $X_{\mathrm{a}}(h)$ and $X_{\mathrm{p}}(h)$ profiles are marked by indices "a" and "p", respectively. All three curves $X_{\mathrm{p}}(h)=$ $X_{\mathrm{p} 1}(h), X_{\mathrm{p} 2}(h), X_{\mathrm{p} 0}(h)$ are coincident in Fig. 4 (left plot). However, some distinction is seen in the right part of Fig. 4, where the altitude dependence of the total absorption is 
shown. This may be connected with influence of the ionosphere. Measured values of the total absorption coincide with a mean value of $\Gamma$ equal to $0.0096 \pm 0.0024 \mathrm{db} \mathrm{km}^{-1}$ and correspond to the MIR-geostationary satellites RO data at the $32 \mathrm{~cm}$ wavelength obtained in monochromatic regime (Pavelyev et al., 1996).

\subsection{Determination of tilt, height, and displacement of inclined layers}

If centre of symmetry does not coincide with the expected location - point $O$, principle of locality states:

$X_{\mathrm{a}}(t) \equiv X_{\mathrm{p}}^{\prime}(t) ; 1-X_{\mathrm{a}}(t)=m^{\prime} a=\frac{m^{\prime}}{m}\left[1-X_{\mathrm{p}}(t)\right]$,

where magnitudes of the refractive attenuation $X_{\mathrm{p}}(t)$ and coefficient $m$ correspond to position of the point $O$. Using relationship (Eq. 1) connecting coefficient $m^{\prime}$ with the distances $d_{1}^{\prime}, d_{2}^{\prime}$, and impact parameter $p_{\mathrm{s}}^{\prime}$, one can obtain

$$
\frac{m^{\prime}}{m}-1=\frac{d_{2}^{\prime} d_{1}^{\prime}\left(\mathrm{d} p_{\mathrm{s}} / \mathrm{d} t\right)^{2}}{d_{2} d_{1}\left(\mathrm{~d} p_{\mathrm{s}}^{\prime} / \mathrm{d} t\right)^{2}}-1 ; d_{1}+d_{2}=d_{1}^{\prime}+d_{2}^{\prime}=R_{0} .
$$

If the displacement of spherical symmetry centre satisfies the following conditions:

$\frac{\mathrm{d} p_{\mathrm{s}}}{\mathrm{d} t} \approx \frac{\mathrm{d} p_{\mathrm{s}}^{\prime}}{\mathrm{d} t} ; \frac{d_{2}}{R_{0}} \ll 1 ; \frac{d_{2}^{\prime}}{R_{0}} \ll 1$,

one can find from Eqs. (6), (7):

$X_{\mathrm{p}}(t)-X_{\mathrm{a}}(t)=\frac{d_{2}^{\prime}-d_{2}}{d_{2}}\left[1-X_{\mathrm{p}}(t)\right]=\frac{d}{d_{2}}\left[1-X_{\mathrm{p}}(t)\right]$,

where $d$ is the distance $D D^{\prime}$ (see Fig. 1). It follows from Eqs. (5), (8) that amplitudes $A_{\mathrm{a}}, A_{\mathrm{p}}$ of variations of the refractive attenuations $-1-X_{\mathrm{a}}(h), 1-X_{\mathrm{p}}(h)$ and distance $d$ are connected by the following equations:

$m^{\prime}=\frac{A_{\mathrm{a}}(t)}{A_{\mathrm{p}}(t)} m ; d=\left[\frac{A_{\mathrm{a}}(t)}{A_{\mathrm{p}}(t)}-1\right] d_{2}$.

The coefficients $m, m^{\prime}$ are slowly changing as functions of time. Therefore the coefficient $m^{\prime}$ and the layer's displacement $d$ can be estimated in the time instant when the amplitude $A_{\mathrm{p}}(t)$ achieves maximal magnitude. This allows finding, if absorption is absent, the displacement $d$ of the tangential point $T^{\prime}$ with respect to the ray perigee $T$ (Fig. 1) as well as the layer's height $h^{\prime}$ and inclination $\delta$ from following equations:

$$
\begin{aligned}
& d=d_{2}^{\prime}-d_{2}=d_{2}(\alpha-1) ; \alpha=\frac{A_{\mathrm{a}}}{A_{\mathrm{p}}} ; d_{2} \\
& =\sqrt{R_{\mathrm{L}}^{2}-p_{\mathrm{s}}^{2}}, h^{\prime}=h+\Delta h, \Delta h=\frac{\mathrm{d} \delta}{2}, \delta=\frac{d}{r_{\mathrm{e}}}, r_{\mathrm{e}}=|T O| .
\end{aligned}
$$

The amplitudes $A_{\mathrm{a}}, A_{\mathrm{p}}$ of variations of the refractive attenuations $1-X_{\mathrm{a}}(h)$ and $1-X_{\mathrm{p}}(h)$, can be evaluated, for example, using the Hilbert numerical transform. The amplitude $A_{\mathrm{p}}(t)$ of refractive attenuation $X_{\mathrm{p}}(t)$ is evaluated using the coefficient $m$ corresponding to the centre of Earth (or another planet) $O$. Depending on the sign of the difference $A_{\mathrm{a}}-A_{\mathrm{p}}$, the value of $d$ is positive (or negative), and point $T^{\prime}$ is located on the $G T$ or $T L$ lines. Note that relationship (Eq. 9) is fulfilled if one of the satellites is much farther away from the centre of symmetry than the other. This condition is usually satisfied during the Earth or planetary RO missions (Fjeldbo, 1964; Yakovlev, 2002).

The spherical symmetry of a medium with new centre $O^{\prime}$ justifies application of the Abel transformation for solution of the inverse problem (Pavelyev et al., 2008a, b). The time derivative of the phase path excess $\Phi(t)$ is used to obtain the temporal dependence of the impact parameter $p^{\prime}$ :

$p^{\prime}-p_{\mathrm{s}}^{\prime}=-m^{\prime} \frac{\mathrm{d} \Phi}{\mathrm{d} t} \frac{\mathrm{d} p_{\mathrm{s}}}{\mathrm{d} t}=-m \alpha \frac{\mathrm{d} \Phi}{\mathrm{d} t} \frac{\mathrm{d} p_{\mathrm{s}}}{\mathrm{d} t}=\alpha\left(p-p_{\mathrm{s}}\right)$.

To solve the inverse problem, the following formulas are used for the Abel transform (Hocke, 1997; Pavelyev et al., 2012) (for simplicity, the bar in designations of the impact parameters $p^{\prime}, p_{\mathrm{s}}^{\prime}$ is deleted):

$N(p)=-\frac{1}{\pi} \int_{p}^{\infty} \ln \left(\frac{x}{p}+\sqrt{\frac{x^{2}}{p^{2}}-1}\right) \frac{\mathrm{d} \xi(x)}{\mathrm{d} x} \mathrm{~d} x$,

where $p$ is the impact parameter corresponding to ray $G T L$ at the instant of time $t$ and $N(p)$ is the refractivity. The vertical gradient of refractivity $\mathrm{d} N(p) / \mathrm{d} h$ can be found from Eq. (12) using a relationship:

$\frac{\mathrm{d} N(p)}{\mathrm{d} h}=\frac{1+N(p)}{1-\frac{\mathrm{d} N(p)}{\mathrm{d} p} r_{T}} \frac{\mathrm{d} N(p)}{\mathrm{d} p}$,

where $r_{T}$ is the distance $T^{\prime} O^{\prime}$ (Fig. 1). Derivative of the bending angle $\xi(p)$ on impact parameter $p$ can be found from the RO signal intensity or the RO phase path excess data (Pavelyev and Kucheryavenkov, 1978; Pavelyev et al., 2012, 2013), i.e.,

$$
\begin{aligned}
& X(p)=\frac{p}{p_{\mathrm{s}}\left|1-\sqrt{R_{2}^{2}-p^{2}} \sqrt{R_{1}^{2}-p^{2}} \frac{\mathrm{d} \xi(p)}{\mathrm{d} p} R_{0}^{-1}\right|} ; \\
& \frac{\mathrm{d} \xi(p)}{\mathrm{d} p}=\left(1-p_{\mathrm{s}} p^{-1} X^{-1}\right) \frac{R_{0}}{\sqrt{R_{2}^{2}-p^{2}} \sqrt{R_{1}^{2}-p^{2}}} \\
& \approx \frac{\left(1-X^{-1}\right) R_{0}}{\sqrt{R_{1}^{2}-p^{2}} \sqrt{R_{2}^{2}-p^{2}}} .
\end{aligned}
$$

The last equation Eq. (14) for $\mathrm{d} \xi(p) / \mathrm{d} p$ is valid under condition: $p \approx p_{\mathrm{s}}$. Substitution Eq. (14) in Eq. (12) gives with accounting for relation $\frac{\mathrm{d} p}{\mathrm{~d} t}=X(p) \frac{\mathrm{d} p_{\mathrm{s}}}{\mathrm{d} t}$ (Kalashnikov et al., 

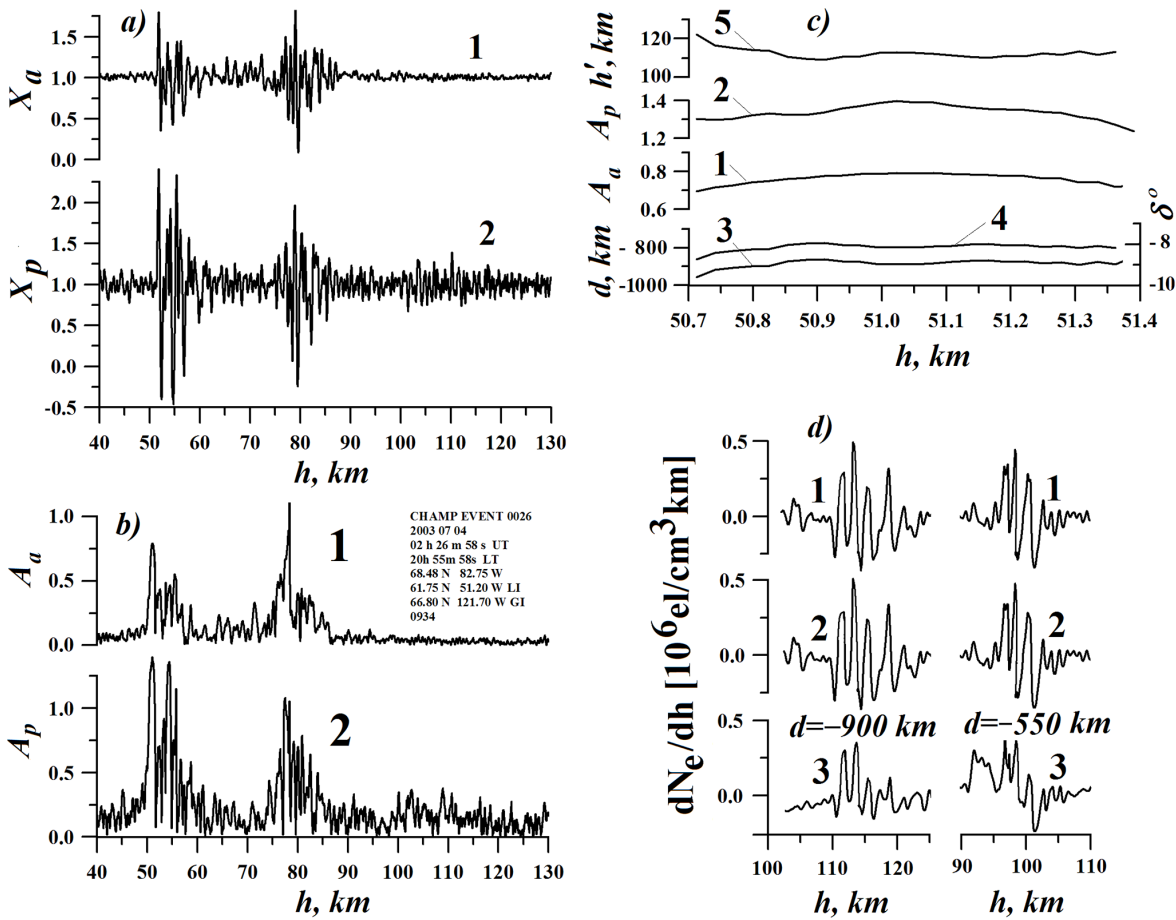

Figure 5. (a) Comparison of the refractive attenuations $X_{\mathrm{a}}(h), X_{\mathrm{p}}(h)$ found from the RO intensity and eikonal data at GPS frequency F1 (curves 1 and 2, respectively). (b) The amplitudes $A_{\mathrm{a}}, A_{\mathrm{p}}$ of analytical signals corresponding to the variations of the refractive attenuations $1-X_{\mathrm{a}}(h)$ and $1-X_{\mathrm{p}}(h)$ (curves 1 and 2), respectively. (c) Location of the first layer using amplitudes $A_{\mathrm{a}}, A_{\mathrm{p}}$. (d) Vertical profiles of the gradients of electron density in the layers.

1986):

$N(p)=\frac{1}{\pi} \int_{-\infty}^{t(p)} \ln \left(\frac{x}{p}+\sqrt{\frac{x^{2}}{p^{2}}-1}\right)$

$\frac{[X(t)-1] R_{0}}{\sqrt{R_{1}^{2}-x(t)^{2}} \sqrt{R_{2}^{2}-x(t)^{2}}} \frac{\mathrm{d} p_{\mathrm{s}}(t)}{\mathrm{d} t} \mathrm{~d} t ;$

$x(t)=p(t) ;-\infty<t \leq t(p)$.

From Eqs. (5), (14), and (15), one can obtain a modernized formula for the Abel inversion, i.e.,

$N(p)=-\frac{1}{\pi} \int_{-\infty}^{t(p)} \ln \left(\frac{x}{p}+\sqrt{\frac{x^{2}}{p^{2}}-1}\right)$

$\frac{m^{\prime} a R_{0}}{\sqrt{R_{1}^{2}-x(t)^{2}} \sqrt{R_{2}^{2}-x(t)^{2}}} \frac{\mathrm{d} p_{\mathrm{s}}(t)}{\mathrm{d} t} \mathrm{~d} t ;$

$x(t)=p(t) ;-\infty<t \leq t(p)$,

where $m^{\prime}$ can be determined from the first equation Eq. (9).

When the position of the spherical symmetry centre is known (for example, a centre of symmetry coincides with the centre of the Earth), Eqs. (15) and (16) are new relationships for solution of the RO inverse problem. Unlike the pre- vious solution (Eq. 12), the Eqs. (15) and (16) do not contain the angle of refraction, and include only temporal dependences of the refractive attenuation $X(t)$, eikonal acceleration, and impact parameter. Note that Eqs. (15) and (16) provide the Abel transform in the time domain where a layer contribution does exist. The linear part of the regular trend due to the influence of the upper ionosphere is removed because the eikonal acceleration $a$ in Eq. (16) contains the second derivative of time. However, the influence of the upper ionosphere exists because it may contribute in the impact parameter $p(t)$. Also, nonlinear contribution of the upper ionosphere remains in the eikonal acceleration $a$. Therefore, Eq. (16) approximately gives that part of the refractivity altitude distribution, which is connected with the influence of a sharp plasma layer. The electron density vertical distribution in the Earth's ionosphere $N_{\mathrm{e}}(h)$ is connected at GPS frequencies with the refractivity $N(h)$ via the following relationship:

$N_{\mathrm{e}}(h)=-\frac{N(h)}{40.3} f^{2}$,

where $f$ is the carrier frequency $[\mathrm{Hz}]$, and $N_{\mathrm{e}}(h)$ is the electron content $\left[\mathrm{el} \mathrm{m}^{-3}\right]$.

Examples of application of Eqs. (12)-(16) for estimation of the location, inclination, and real height of ionospheric layers are given in Fig. 5. To consider a possibility to locate the plasma layers, a CHAMP RO event 026 (04 July, 
2003, 02:27 UT; geographic coordinates $68.5^{\circ} \mathrm{N} 82.8^{\circ} \mathrm{W}$ ) with strong quasi-regular intensity and phase variations is used. The refractive attenuations of the CHAMP RO signals $X_{\mathrm{a}}(h), X_{\mathrm{p}}(h)$ found from the RO signal intensity and eikonal data are shown in Fig. 5a as functions of the RO ray perigee altitude $h$. The eikonal acceleration $a$ has been estimated by double differentiation of a second-power least square polynomial over a sliding time interval $\Delta t=0.5 \mathrm{~s}$. This time interval approximately corresponds to the vertical size of Fresnel zone of about $1 \mathrm{~km}$ since the vertical component of the RO radio ray was about $2.1 \mathrm{~km} \mathrm{~s}^{-1}$. The refractive attenuation $X_{\mathrm{p}}(h)$ is derived from the evaluated magnitude $a$ using Eq. (1), and $m$ value is obtained from the orbital data. The refractive attenuation $X_{\mathrm{a}}(h)$ is derived from the RO signal intensity data by a sliding least-square polynomial having the same power with averaging in the time interval of $0.5 \mathrm{~s}$. In the altitude ranges of 50-60 and $75-85 \mathrm{~km}$, the refractive attenuations variations $X_{\mathrm{a}}(h)$ and $X_{\mathrm{p}}(h)$ are strongly connected and may be considered as coherent oscillations caused by sporadic layers (Fig. 5a, curves 1 and 2). Using a Hilbert numerical transform, the amplitudes $A_{\mathrm{a}}, A_{\mathrm{p}}$ of analytical signals related to $1-X_{\mathrm{a}}(h)$ and $1-X_{\mathrm{p}}(h)$ have been computed and are shown in Fig. 5b, curves 1 and 2, respectively. In the altitude range of 50-60 km, amplitudes $A_{\mathrm{a}}, A_{\mathrm{p}}$ are nearly identical, but the magnitude of $A_{\mathrm{a}}$ is about 1.7 times below than that of $A_{\mathrm{p}}$. Accordingly, the displacement $d$ found from Eq. (9) is negative, and a plasma layer is displaced from the RO ray perigee $T$ in the direction to satellite $L$ (see Fig. 1). A similar form of variations of the refractive attenuations $1-X_{\mathrm{a}}(h)$ and $1-X_{\mathrm{p}}(h)$ allows locating the detected ionospheric layer. Displacement $d$ corresponding to a plasma layer recorded at the $51 \mathrm{~km}$ altitude of the RO ray perigee is shown in Fig. 5c. Curves 1 and 2 in Fig. 5c correspond to amplitudes $A_{\mathrm{a}}$ and $A_{\mathrm{p}}$, respectively. Curve 3 describes the displacement $d$ found using Eq. (9) from amplitudes $A_{\mathrm{a}}, A_{\mathrm{p}}$ in the $50.7-51.4 \mathrm{~km}$ altitude interval. Changes in $d$ are concentrated in the altitude range of -900 to $-950 \mathrm{~km}$ when the functions $A_{\mathrm{a}}, A_{\mathrm{p}}$ vary near their maximal values of 0.75 and 1.36 in the ranges of $0.7 \leq A_{\mathrm{a}} \leq 0.75$; $1.29 \leq A_{\mathrm{p}} \leq 1.36$, respectively. The statistical error in the determination of ratio $\frac{A_{\mathrm{a}}(t)}{A_{\mathrm{p}}(t)}$ in Eq. (9) is minimal when $A_{\mathrm{p}}(t)$ is maximal. If the relative error in the measurements of $A_{\mathrm{p}}$ is $5 \%$, according to Fig. $5 \mathrm{c}$, the accuracy in the estimation of $d$ is about $\pm 120 \mathrm{~km}$. The inclination of a plasma layer to a local horizontal direction $\delta$ calculated using Eq. (10) is approximately equal to $\delta=8.2^{\circ} \pm 0.2^{\circ}$. The vertical gradient $\frac{\mathrm{d} N_{\mathrm{e}}(h)}{\mathrm{d} h}$ of electron density distribution $N_{\mathrm{e}}(h)$ for the given RO event is shown in Fig. 5d. Curves 1 and 2 correspond to the vertical gradient $\frac{\mathrm{d} N_{\mathrm{e}}(h)}{\mathrm{d} h}$ retrieved using Eqs. (12) and (16), respectively. Curve 3 is related to the vertical gradient $\frac{\mathrm{d} N_{\mathrm{e}}(h)}{\mathrm{d} h}$ retrieved using the refractive attenuation $X_{\mathrm{a}}(h)$ and formula (Eq. 15). The real altitude of the ionospheric layers is indicated on the horizontal axis in Fig. 5d. Two ionospheric layers are seen (curves 1, 2, and 3 in Fig. 5d). The first layer is located on line $T L$ at the 110 to $120 \mathrm{~km}$ altitudes at a distance of $900 \mathrm{~km}$ from point $T$ (Fig. $5 \mathrm{~d}$, left). The second layer is located near the RO perigee at the 95 to $105 \mathrm{~km}$ altitudes (Fig. 5d, right). From comparison of the refractive variations $X_{\mathrm{a}}(h)$ and $X_{\mathrm{p}}(h)$ (Fig. 5a, curves 1 and 2) and vertical gradients of the electron content (Fig. 5d), the width of sporadic E-layers is nearly equal to the altitude interval of intensity variations of the RO signals. From Fig. 5d, variations of the vertical gradient of electron density are concentrated in the interval $-0.4 \times 10^{6}\left[\mathrm{elcm}^{-3} \mathrm{~km}^{-1}\right]<\frac{\mathrm{d} N_{\mathrm{e}}(h)}{\mathrm{d} h}<0.5 \times 10^{6}$ [elcm ${ }^{-3} \mathrm{~km}^{-1}$ ]. These magnitudes of $N_{\mathrm{e}}(h)$ are typical for sporadic E-layers (Kelley and Heelis, 2009). The height interval of the RO signal intensity variations is nearly equal to the height interval of variations in the electron density and its gradient. It follows that the standard definition of perigee of radio ray in the RO method as a minimal value of the distance of the ray path to the surface leads to an underestimation (bias) of layers altitude in the atmosphere (ionosphere) of Earth (or another planet). This error is zero for horizontal layers and increases with their inclination.

\subsection{Separation of layers and small-scale irregularities contributions in the RO signal}

The principle of locality allows one to separate the contributions of layers and irregular inhomogeneities in the RO signal. According to identity (5), the coherent and incoherent components of the RO atmospheric signal $C(h), I(h)$ due to the layers and irregularities influence can be estimated as follows:

$$
\begin{aligned}
& C(h)=\left[X_{\mathrm{a}}(h)+X_{\mathrm{p}}(h)\right] / 2-P(h) ; I(h) \\
& \quad=\left[X_{\mathrm{a}}(h)-X_{\mathrm{p}}(h)\right] / 2,
\end{aligned}
$$

where $P(h)$ is the polynomial approximation describing main atmospheric contribution in the RO signal. If location of the spherical symmetry centre is known, $P(h)$ should be the same for the refractive attenuations $X_{\mathrm{a}}(h), X_{\mathrm{p}}(h)$. Example of separation of the layers and inhomogeneities contributions in the RO signal is presented below. Figure 6 shows altitude dependences of the refractive attenuations $X_{\mathrm{a}}(h), X_{\mathrm{p}}(h)$ for the CHAMP RO event 7 April 2003 carried out at 02:34 UT in the area with geographical coordinates $2.5^{\circ} \mathrm{S} 291.7^{\circ} \mathrm{W}$. Vertical profiles of the refractive attenuations $X_{\mathrm{a}}(h), X_{\mathrm{p}}(h)$ reveal the coherent variations of $X_{\mathrm{a}}(h), X_{\mathrm{p}}(h)$ indicating contribution of the atmospheric layers (Fig. 6, left, top, curves $X_{\mathrm{A}}, X_{\mathrm{P}}$ are displaced for comparison). Dotted curves in Fig. 6, left (displaced for comparison), describe polynomial approximations $P(h)$ of the slow changing altitude dependences of $X_{\mathrm{a}}(h), X_{\mathrm{p}}(h)$. The coherent and incoherent parts of the RO signal $C(h), I(h)$ are obtained using formulas (Eq. 18) and are shown in the Fig. 6, left, bottom plot (curves 1 and 2, respectively). Intensity of the coherent component $C(h)$ by an order of magnitude prevails the corresponding value of $I(h)$, thus, indicating importance of the layers contribution in the RO signal. Usually this 

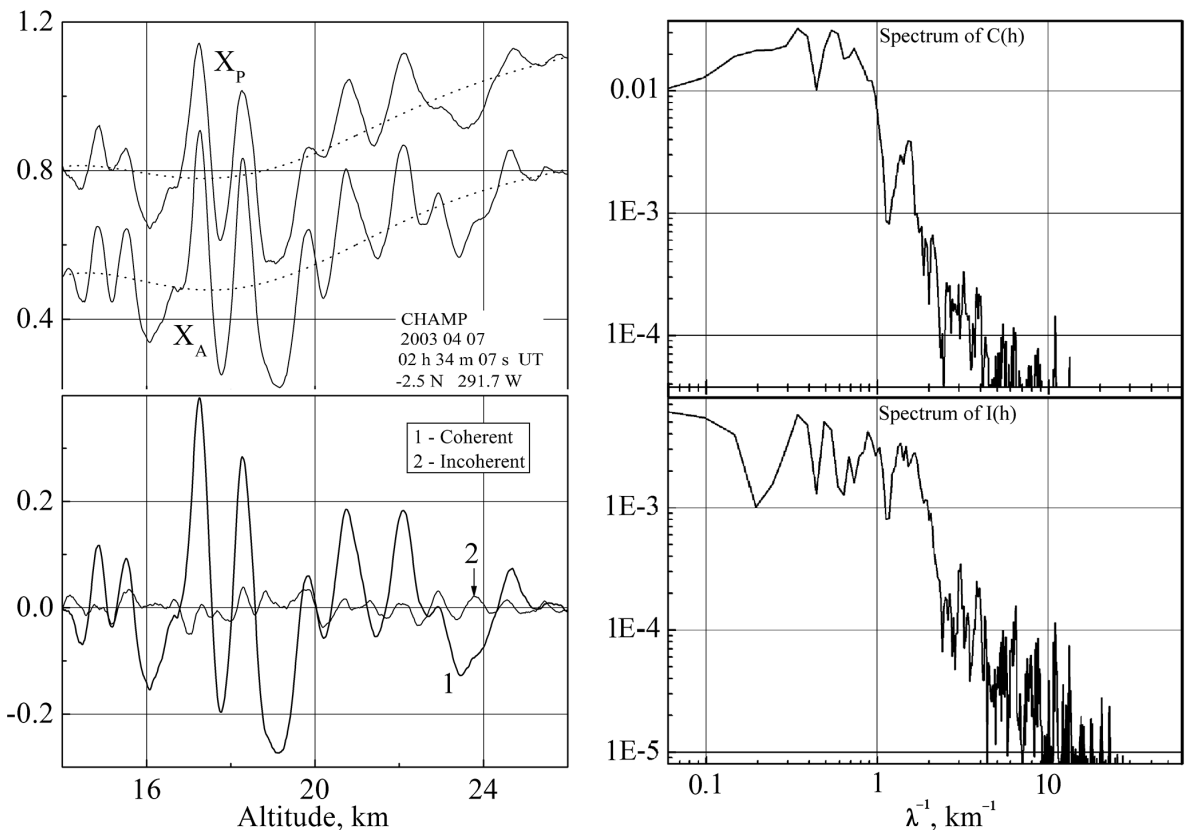

Figure 6. Left, top - Comparison of the refractive attenuations $X_{\mathrm{a}}(h), X_{\mathrm{p}}(h)$ found from the RO intensity and eikonal data at GPS frequency $\mathrm{F} 1$ (curves $X_{\mathrm{A}}$ and $X_{\mathrm{P}}$, respectively, displaced for comparison). Dotted curves show the polynomial approximations of the refractive attenuations $X_{\mathrm{a}}(h), X_{\mathrm{p}}(h)$. Left, bottom - Contribution of the atmospheric layers (coherent component of the RO signal) and small scale irregularities (incoherent component) (curves 1 and 2, respectively). Right - Spatial spectra of the coherent and incoherent components of the RO signal (top and bottom panels, respectively).

contribution may be assigned to effects of small-scale irregularities (Cornman et al., 2012). An analysis of spatial spectra of coherent and incoherent components is presented in the Fig. 6, right, top and bottom plots, respectively. The forms of spectra $C(h), I(h)$ are similar in the interval of the vertical periods greater than $1 \mathrm{~km}$. In the interval below $1 \mathrm{~km}$, the power degrees of spectra inclination are different and equal to $3.7 \pm 0.2$ and $2.1 \pm 0.2$ for components $C(h)$ and $I(h)$, respectively. This indicates a different origin of the components $C(h)$ and $I(h)$. The inclination of the $I(h)$ spectrum is nearly $8 / 3$ which corresponds to contribution of the turbulent irregularities in variations of the RO signal intensity (Gurvich and Yakushkin, 2004). The value 3.7 corresponding to the coherent component $C(h)$ is near the inclination of spatial spectrum of anisotropic internal gravity waves in accordance with the theory developed by Gurvich and Chunchuzov (2008).

Parameters of coherent and incoherent components introduced in the Table 1 illustrate a possibility to separate the contributions of atmospheric layers and turbulent structures in the RO signal. The time and geographic coordinates are shown in the first two columns of Table 1 . The rms deviations $\sigma_{\mathrm{A}}, \sigma_{\mathrm{P}}$ of the refractive attenuations $X_{\mathrm{a}}(h), X_{\mathrm{p}}(h)$ and dispersion of the components $C(h)$ and $I(h) \sigma_{\mathrm{c}}, \sigma_{\text {in }}$ are presented in the next four columns. The altitude interval $h_{\mathrm{b}} / h_{\mathrm{u}}$ of measurements of $\sigma_{\mathrm{A}}, \sigma_{\mathrm{P}} ; \sigma_{\mathrm{c}}, \sigma_{\mathrm{in}}$; and correlation coefficient $r_{\mathrm{c}}$ between the refractive attenuations variations $X_{\mathrm{A}}(h)$, $X_{\mathrm{P}}(h)$ are indicated in the last two columns of Table 1 . As a rule the rms deviation $\sigma_{\mathrm{c}}$ of the coherent component $C(h)$ is greater than that one of incoherent part $I(h) \sigma_{\text {in }}$ by a factor of 4-5. This indicates on the prevailing contribution of the atmospheric layers as compared with influence of the irregularities in the RO signal. High level of correlation $r_{\mathrm{c}}$ (in the interval 0.84-0.96) between the variations of the refractive attenuations $X_{\mathrm{a}}(h), X_{\mathrm{p}}(h)$ indicates the practical and theoretical importance of the locality principle for separation of the atmospheric layers and irregularities in the RO signal at a single frequency.

\section{Relationships between eikonal variations and scintillations index $\mathrm{S4}$}

According to the locality principle, a global correlation between the phase and intensity variations of the RO signal can exist. Index S4 (I), as measured from intensity variations, should be correlated with index S4 (F), defined by the second derivative of time of the eikonal of the RO signal at GPS frequencies F1 and F2. According to the principle of locality in the case of spherical symmetric medium, the following connections can exist: 
Table 1. Parameters of coherent and incoherent components.

\begin{tabular}{llllllll}
\hline Time (UT) & Location & $\sigma_{\mathrm{A}}$ & $\sigma_{\mathrm{P}}$ & $\sigma_{\mathrm{c}}$ & $\sigma_{\text {in }}$ & $h_{\mathrm{b}} / h_{\mathrm{u}}, \mathrm{km}$ & $r_{\mathrm{c}}$ \\
\hline $2003041721: 38$ & $58.9^{\circ} \mathrm{N} 117.5^{\circ} \mathrm{E}$ & 0.084 & 0.062 & 0.071 & 0.018 & $12 / 30$ & 0.88 \\
$2003041722: 05$ & $45.8^{\circ} \mathrm{S} 146.9^{\circ} \mathrm{E}$ & 0.07 & 0.058 & 0.062 & 0.015 & $14 / 25$ & 0.88 \\
$2003041723: 13$ & $48.7^{\circ} \mathrm{N} 124.4^{\circ} \mathrm{E}$ & 0.11 & 0.097 & 0.104 & 0.022 & $12 / 27$ & 0.91 \\
$2003042600: 06$ & $55.8^{\circ} \mathrm{N} 74.2^{\circ} \mathrm{E}$ & 0.065 & 0.052 & 0.056 & 0.016 & $11 / 26$ & 0.85 \\
$2003042600: 13$ & $31.1^{\circ} \mathrm{N} 74.6^{\circ} \mathrm{E}$ & 0.051 & 0.036 & 0.042 & 0.011 & $18 / 32$ & 0.86 \\
$2003042601: 21$ & $51.8^{\circ} \mathrm{N} 125.2^{\circ} \mathrm{W}$ & 0.096 & 0.076 & 0.085 & 0.015 & $12 / 26$ & 0.94 \\
$2003042601: 23$ & $58.1^{\circ} \mathrm{N} 92.8^{\circ} \mathrm{W}$ & 0.055 & 0.039 & 0.046 & 0.011 & $13 / 25$ & 0.88 \\
$2003042604: 42$ & $65.3^{\circ} \mathrm{N} 35.3^{\circ} \mathrm{E}$ & 0.056 & 0.043 & 0.048 & 0.014 & $10 / 22$ & 0.85 \\
$2003050300: 34$ & $0.4^{\circ} \mathrm{S} 63.0^{\circ} \mathrm{E}$ & 0.11 & 0.086 & 0.096 & 0.027 & $17.5 / 26$ & 0.86 \\
$2003050301: 27$ & $22.2^{\circ} \mathrm{N} 121.6^{\circ} \mathrm{W}$ & 0.14 & 0.11 & 0.123 & 0.023 & $12 / 27$ & 0.94 \\
$2003050303: 35$ & $12.6^{\circ} \mathrm{N} 38.6^{\circ} \mathrm{E}$ & 0.079 & 0.065 & 0.071 & 0.012 & $20 / 30$ & 0.95 \\
$2003050304: 56$ & $59.4^{\circ} \mathrm{N} 17.0^{\circ} \mathrm{E}$ & 0.05 & 0.039 & 0.043 & 0.011 & $12.5 / 26.5$ & 0.87 \\
$2003041207: 48$ & $45.6^{\circ} \mathrm{S} 7.6^{\circ} \mathrm{E}$ & 0.066 & 0.047 & 0.056 & 0.012 & $14 / 25$ & 0.915 \\
$2003041208: 25$ & $7.4^{\circ} \mathrm{N} 153.9^{\circ} \mathrm{E}$ & 0.103 & 0.076 & 0.088 & 0.013 & $18 / 30$ & 0.96 \\
$2003041208: 53$ & $57.5^{\circ} \mathrm{N} 42.2^{\circ} \mathrm{W}$ & 0.06 & 0.052 & 0.055 & 0.011 & $12 / 24$ & 0.918 \\
$2003041209: 37$ & $70.8^{\circ} \mathrm{S} 165.0^{\circ} \mathrm{E}$ & 0.069 & 0.054 & 0.06 & 0.015 & $12 / 22$ & 0.88 \\
$2003041210: 08$ & $52.2^{\circ} \mathrm{N} 150.9^{\circ} \mathrm{E}$ & 0.085 & 0.069 & 0.075 & 0.016 & $12.5 / 27$ & 0.92 \\
\hline
\end{tabular}
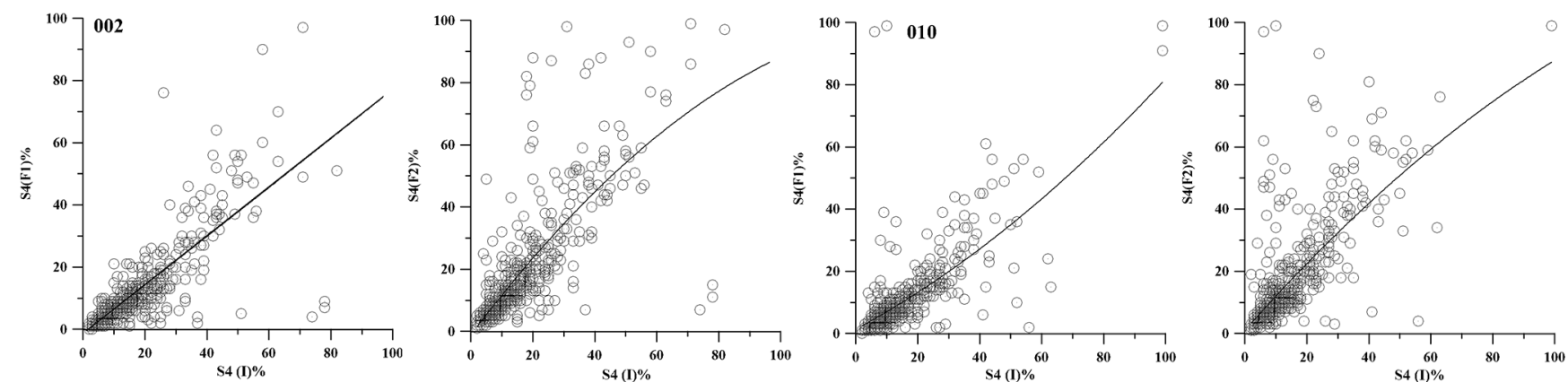

Figure 7. Correlation of index S4(I) measured from the intensity variations of the GPS RO signal at frequency F1 and parameters S4(F1) and S4(F2) found from the eikonal variations at GPS frequencies F1 and F2.

$$
\begin{aligned}
& \mathrm{S} 4\left(X_{\mathrm{a}}\right)=\sqrt{\frac{\left\langle X_{\mathrm{a}}^{2}\right\rangle-\left\langle X_{\mathrm{a}}\right\rangle^{2}}{\left\langle X_{\mathrm{a}}\right\rangle^{2}}} ; \mathrm{S} 4\left(X_{\mathrm{p}}\right)=\sqrt{\frac{\left\langle X_{\mathrm{p}}^{2}\right\rangle-\left\langle X_{\mathrm{p}}\right\rangle^{2}}{\left\langle X_{\mathrm{p}}\right\rangle^{2}}} ; \\
& \mathrm{S} 4\left(X_{\mathrm{a}}\right) \equiv \mathrm{S} 4\left(X_{\mathrm{p}}\right) .
\end{aligned}
$$

Figures 7 and 8 show the results of correlation of index $\mathrm{S} 4(\mathrm{I})$, defined by the variations of the intensity I at the frequency F1 with indices S4(F1), S4(F2) measured from the second derivative of the phase paths excess at frequencies F1, F2 during FORMOSAT-3 RO events held in January and February 2012. Circles in Figs. 7 and 8 correspond to the experimental values of index S4 (I) (vertical axis) and S4 (F1), S4(F2) (horizontal axis), respectively. The solid curves in Figs. 7 and 8 are regression lines and have been found by the least squares method. The correlation coefficient of index S4(I) to S4(F1) and S4(F2) varies in the intervals 0.69 to 0.78 and $0.70-0.75$, respectively. The correlation coefficient of index S4(I) with combined index [S4(F1)+S4(I)]/2 is very high and changes in the interval 0.91-0.97. Measured correlation values indicate a significant contribution of regular layers in ionospheric variations of the intensity and phase of the RO signals at frequencies F1, F2. High correlation between variations of the indices S4(I), S4(F1), and S4(F2) indicates substantially lower influence of the small-scale irregularities on the RO signal as compared with contribution of layered structures in the ionosphere.

\section{Conclusions}

The principle of locality is a key regularity that extends applicable domain of the RO method, widens possibilities and opens new directions of the $\mathrm{RO}$ geophysical applications to remote radio sensing the atmosphere and ionosphere of the Earth and other planets. These directions include: (i) innovative estimating the altitude dependence of the total absorption of radio waves using the $\mathrm{RO}$ amplitude and phase variations at a single frequency; (ii) evaluation of the slope, altitude, and 

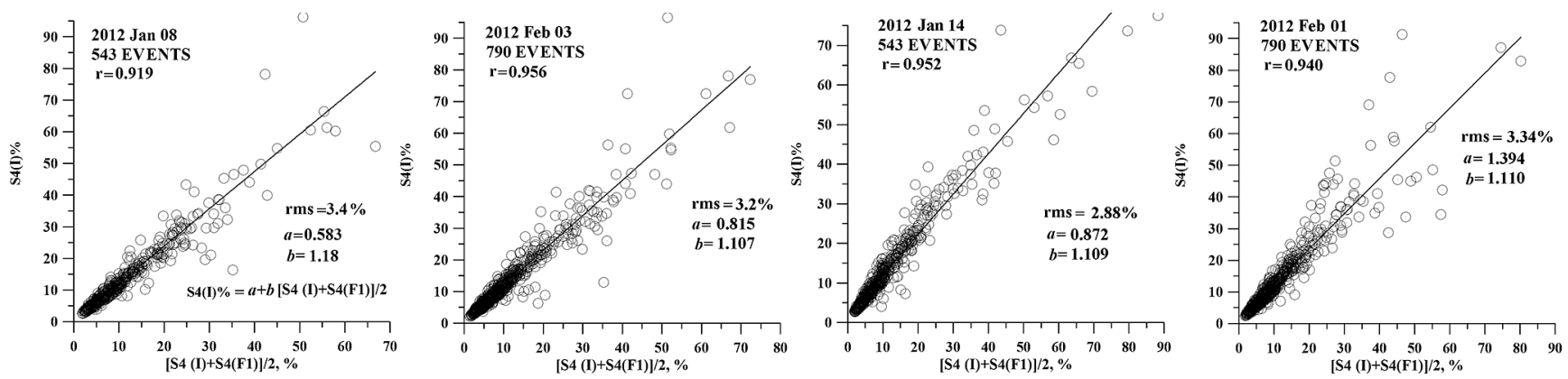

Figure 8. Correlation of indices S4(I) and [S4(F1)+S4(I)]/2.

horizontal displacement of the atmospheric and ionospheric layers from the RO signal intensity and phase data using the eikonal acceleration/intensity technique; (iii) separation of layers and irregularities contributions in the RO signal, determination of vertical profiles of the turbulent and small-scale structures by joint analysis of the RO signal eikonal and intensity variations; and (iv) introduction of the new combined phase-intensity index for the RO study of multilayered structures and wave processes. This regularity is valid for every $\mathrm{RO}$ ray trajectory in geometrical optics approximation including reflections from the surface.

As follow from Sect. 3.1 the total absorption is stronger in the equatorial region than at high latitudes, pointing to the role of water vapour and, possible, the clouds of liquid water, ice and snow. The contribution of the clouds water (fog, rain, and hydrometeors) in the RO signal should be analyzed separately in future investigations. First of all, the theory of radio waves propagation should be reconsidered for the case when the radio waves are propagating along the clouds under different temperature conditions.

Mass-scale measurements of the total absorption at the altitude below $15 \mathrm{~km}$ depend on the quality of the GPS receivers onboard of the RO missions. The total absorption measurements are possible only in the case when the low and high frequency noise are small enough for coinciding of the polynomial approximation of the RO intensity and phase data at the altitudes between $15-60 \mathrm{~km}$. Also the stability of the RO signal phase data and accuracy of the total absorption measurements are determined by precision of the open-loop regime of the GPS RO receivers below $8 \mathrm{~km}$ altitude. Analysis of these technological aspects of the RO measurements is the task of future works.

It follows from Sect. 3.2 that RO definition of the vertical location of layers as coinciding with the altitude of the radio ray perigee can lead to an underestimation of their height in the atmosphere (ionosphere) of Earth and other planets. This systematic bias is zero for horizontal layers and strongly increases with their inclination in the range $1-10^{\circ}$ from $1 \mathrm{~km}$ up to about of several dozen kilometres. The measured inclination of layers can be applied for estimating the orientation of wave fronts in the ionosphere (or atmosphere) and may be used for determination of the important parameters of internal waves including the internal frequency, direction and magnitude of kinetic momentum. A new method of estimating the electron density distribution in plasma layers (described in Sect. 3.2) should be a subject of future comparison with the ionosondes and tomographic data.

Mass-scale measurements of coherent and incoherent component of the RO signal (Sect. 3.3) and introduced (Sect. 4) combined phase-intensity ionospheric index are important for investigation of the temporal, seasonal and regional evolution of the layered and turbulent structures at different altitudes in the ionosphere and atmosphere with a global coverage and can be provided in near future with usage of extended volume of the RO data obtained during 20 years (1995-2015) of experimental researches.

Acknowledgements. We are grateful to Taiwan Centre for Space and Remote Sensing Research for access to the FORMOSAT-3 RO data. This work was supported in part by program no. 9 of the Presidium of the Russian Academy of Sciences (RAS), program IV.13 of the Physical Sciences Division RAS, and grant No. 13-02-00526-a from Russian Foundation of Basic Research. We are grateful to R. R. Salimzyanov for help in preparing the manuscript. We send special thanks to the referees for their fruitful remarks and suggestions which helped us to improve our paper.

Edited by: J. Y. Liu

\section{References}

Anthes, R. A.: Exploring Earth's atmosphere with radio occultation: contributions to weather, climate and space weather, Atmos. Meas. Tech., 4, 1077-1103, doi:10.5194/amt-4-1077-2011, 2011.

Arras, C., Wickert, J., Jacobi, Ch., Heise, S., Beyerle, G., and Schmidt, T.: A global climatology of ionospheric irregularities derived from GPS radio occultation, Geophys. Res. Lett., 35, L14809, doi:10.1029/2008GL034158, 2008.

Arras, C., Jacobi, C., Wickert, J., Heise, S., and Schmidt, T.: Sporadic $\mathrm{E}$ signatures revealed from multi-satellite radio occultation measurements, Adv. Radio Sci., 8, 225-230, doi:10.5194/ars-8225-2010, 2010. 
Bai, W. H., Sun, Y. Q., Du, Q. F., Yang, G. L., Yang, Z. D., Zhang, P., Bi, Y. M., Wang, X. Y., Cheng, C., and Han, Y.: An introduction to the FY3 GNOS instrument and mountain-top tests, Atmos. Meas. Tech., 7, 1817-1823, doi:10.5194/amt-7-1817-2014, 2014.

Benzon, H.-H., Nielsen, A. S., and Olsen, L.: An atmospheric wave optics propagator - theory and application, DMI, Scientific Report 03-01, DMI, Copenhagen, Denmark, 1-96, DMI, available at: http://www.dmi.dk/fileadmin/Rapporter/SR/sr03-01.pdf (last access: July 2015), 2003.

Beyerle, G. and Hocke, K.: Observation and simulation of direct and reflected GPS signals in Radio Occultation Experiments, Geophys. Res. Lett., 28, 1895-1898, 2001.

Beyerle, G., Wickert, J., Galas, R., Hocke, K., Konig, R., Marquardt, C., Pavelyev, A. G., Reigber, C., and Schmidt, T.: GPS occultation measurements with GPS/MET and CHAMP, Taikiken Shinpojiumu, 15, 44-47, 2001.

Beyerle, G., Hocke, K., Wickert, J., Schmidt, T., and Reigber, C.: GPS radio occultations with CHAMP: a radio holographic analysis of GPS signal propagation in the troposphere and surface reflections, J. Geophys. Res., 107, 4802, doi:10.1029/2001JD001402, 2002.

Cornman, L. B., Goodrich, R. K., Axelrad, P., and Barlow, E.: Progress in turbulence detection via GNSS occultation data, Atmos. Meas. Tech., 5, 789-808, doi:10.5194/amt-5-789-2012, 2012.

Fjeldbo, G.: Bistatic-Radar Methods for Studying Planetary Ionospheres and Surfaces, Ph.D. thesis, Stanford University, USA, 1964.

Fjeldbo, G., Kliore, A. J., and Eshleman, V. R.: The neutral atmosphere of Venus as studied with the Mariner V radio occultation experiments, Astron. J., 76, 123-140, 1971.

Foelsche, U., Kirchengast, G., Steiner, A. K., Kornblueh, L., Manzini, E., and Bengtsson, L.: An observing system simulation experiment for climate monitoring with GNSS radio occultation data: Setup and test bed study, J. Geophys. Res., 113, D11108, doi:10.1029/2007JD009231, 2008.

Fong, C.-J., Shiau, W.-T., Lin, C.-T., Kuo, T.-C., Chu, C.-H., Yang, S.-K., Nick, L. Y., Chen, S.-S., Kuo, Y.-H., Liou, Y.-A., and Chi, S.: Constellation deployment for the FORMOSAT-3/COSMIC mission, IEEE T. Geosci. Remote Sens., 46, 3367-3379, 2008.

Gorbunov, M. E.: Ionospheric correction and statistical optimization of radio occultation data, Radio Sci., 37, 17-1-17-9, 2002a.

Gorbunov, M. E.: Canonical transform method for processing GPS radio occultation data in lower troposphere, Radio Sci., 37, 9-19-10, doi:10.1029/2000RS002592, 2002b.

Gorbunov, M. E. and Gurvich, A. S.: Microlab-1 experiment: multipath effects in the lower troposphere, J. Geophys. Res., 103, 13819-13826, 1998a.

Gorbunov, M. E. and Gurvich, A. S.: Algorithms of inversion of Microlab-1 satellite data including effects of multipath propagation, Int. J. Remote Sens., 19, 2283-2300, 1998b.

Gorbunov, M. E. and Kirchengast, G.: Processing X/K Band Radio Occultation Data in Presence of Turbulence, Radio Sci., 40, RS6001, doi:10.1029/2005RS003263, 2005.

Gorbunov, M. E. and Lauritsen, K. B.: Analysis of wave fields by Fourier integral operators and its application for radio occultations, Radio Sci., 39, RS4010, doi:10.1029/2003RS002971, 2004.
Gorbunov, M. E., Gurvich, A. S., and Bengtsson, L.: Advanced Algorithms of Inversion of GPS/MET Satellite Data and Their Application to Reconstruction of Temperature and Humidity, report No. 211, Max-Planck-Institute for Meteorology, Hamburg, 40 pp., 1996.

Gorbunov, M. E., Gurvich, A. S., and Shmakov, A. V.: Backpropagation and radio-holographic methods for investigation of sporadic ionospheric E-layers from Microlab-1 data, Int. J. Remote Sens., 23, 675-685, 2002.

Gorbunov, M. E., Lauritsen, K. B., and Leroy, S. S.: Application of Wigner distribution function for analysis of radio occultations, Radio Sci., 45, RS6011, doi:10.1029/2010RS004388, 2010.

Gubenko, V. N., Andreev, V. E., and Pavelyev, A. G.: Detection of layering in the upper cloud layer of Venus northern polar atmosphere observed from radio occultation data, J. Geophys. Res., 113, E03001, doi:10.1029/2007JE002940, 2008a.

Gubenko, V. N., Pavelyev, A. G., and Andreev, V. E.: Determination of the intrinsic frequency and other wave parameters from a single vertical temperature or density profile measurement, J. Geophys. Res., 113, D08109, doi:10.1029/2007JD008920, 2008 b.

Gubenko, V. N., Pavelyev, A. G., Salimzyanov, R. R., and Pavelyev, A. A.: Reconstruction of internal gravity wave parameters from radio occultation retrievals of vertical temperature profiles in the Earth's atmosphere, Atmos. Meas. Tech., 4, 2153-2162, doi:10.5194/amt-4-2153-2011, 2011.

Gurvich, A. S. and Chunchuzov, I. P.: Model of the ThreeDimensional Spectrum of Anisotropic Temperature Irregularities in a Stably Stratified Atmosphere, Izv. Atmos. Ocean. Phys., 44, 567-582, 2008.

Gurvich, A. S. and Krasil'nikova, T. G.: Navigation satellites for radio sensing of the Earth's atmosphere, Sov. J. Remote Sens., 6, 89-93, 1987 (in Russian), 6, 1124-1131, 1990 (in English).

Gurvich, A. S. and Yakushkin, I. G.: Observation of quasiperiodical structures in the strato-sphere from space, Izv. Atmos. Ocean. Phys., 40, 737-746, 2004.

Hajj, G. A. and Romans, L. J.: Ionospheric electron density profiles obtained with the Global Positioning System: results from GPS/MET experiment, Radio Sci., 33, 175-190, 1998.

Hajj, G. A., Ao, C. O., Iijima, B. A., Kuang, D., Kursinski, E. R., Mannucci, A. J., Meehan, T. K., Romans, L. J., de la Torre Juarez, M., and Yunck, T. P.: CHAMP and SAC-C atmospheric occultation results and intercomparisons, J. Geophys. Res., 109, D06109, doi:10.1029/2003JD003909, 2004.

Hinson, D. P., Flasar, F. M., Schinder, A., Twicken, J. D., and Herrera, R. G.: Jupiter's ionosphere: results from the first Galileo radio occultation experiment, Geophys. Res. Lett., 24, 2107-2110, 1997.

Hinson, D. P., Simpson, R. A., Twicken, J. D., Tyler, G. L., and Flasar, F. M.: Initial results from radio occultation measurements with Mars Global Surveyor, J. Geophys. Res., 104, $26997-$ 27012, 1999.

Hocke, K.: Inversion of GPS meteorology data, Ann. Geophys., 15, 443-450, doi:10.1007/s00585-997-0443-1, 1997.

Hocke, K., Pavelyev, A., Yakovlev, O., Barthes, L., and Jakowski, N.: RO data analysis by radio holographic method, J. Atmos. Sol.-Terr. Phys., 61, 1169-1177, 1999.

Igarashi, K., Pavelyev, A. G., Hocke, K., Kucherjavenkov, A. I., Matugov, S. S., Yakovlev, O. I., Pavelyev, D., and Zakharov, A.: Radio holographic principle for observing natural processes in 
the atmosphere and retrieving meteorological parameters from radio occultation data, Earth Planets Space, 52, 868-875, 2000.

Igarashi, K., Pavelyev, A. G., Hocke, K., Pavelyev, D., and Wickert, J.: Observation of wave structures in the upper atmosphere by means of radio holographic analysis of the RO data, Adv. Space Res., 27, 1321-1327, 2001.

Jensen, A. S., Lohmann, M., Benzon, H.-H., and Nielsen, A. S.: Full spectrum inversion of radio occultation signals, Radio Sci., 38, 1040, doi:10.1029/2002RS002763, 2003.

Jensen, A. S., Lohmann, M., Nielsen, A. S., and Benzon, H.-H.: Geometrical optics phase matching of radio occultation signals, Radio Sci., 39, RS3009, doi:10.1029/2003RS002899, 2004.

Jakowski, N., Leitinger, R., and Angling, M.: Radio occultation techniques for probing the ionosphere, Ann. Geophys.-Italy, 47, 1049-1066, 2004.

Joo, S., Eyre, J., and Marriott, R.: The Impact of METOP and Other Satellite Data Within the Met Office Global NWP System Using an Adjoint-Based Sensitivity Method, Forecasting Research Technical Report no. 562, February, 1-18, 2012.

Kalashnikov, I. E., Matyugov, S. S., Pavelyev, A. G., and Yakovlev, O. I.: Analysis of the features of radio occultation method for the Earth's atmosphere study, in: The Book Electromagnetic Waves in the Atmosphere and Space, Nayka Ed., Moscow, 208-218, 1986 (in Russian).

Karayel, E. T. and Hinson, D. P.: Sub-Fresnel vertical resolution in atmospheric profiles from radio occultation, Radio Sci., 32, 411418, 1997.

Kelley, M. C. and Heelis, R. A.: The Earth's Ionosphere: Plasma Physics and Electrodynamics, Elsevier Science, New York, 2009.

Kirchengast, G., Steiner, A. K., Foelsche, U., Kornblueh, L., Manzini, E., and Bengtsson, L.: Spaceborne climate change monitoring by GNSS occultation sensors, in: Proc. 11th Symp. Global Change Studies, AMS Ann. Meeting 2000, Long Beach, Calif., 62-65, 2000.

Kunitsyn, V. E. and Tereshchenko, E. D.: Ionospheric Tomography, Springer-Verlag, Berlin, 2003.

Kunitsyn, V. E., Nesterov, I., Padokhin, A., and Tumanova, Y.: Ionospheric radio tomography based on the GPS/GLONASS navigation systems, J. Commun. Technol. El., 56, 1269-1281, 2011.

Kunitsyn, V. E., Andreeva, E., Nesterov, I., and Padokhin, A.: Ionospheric sounding and tomog-raphy by GNSS, in: Geodetic Sciences - Observations, Modeling and Applications, chapter 6, edited by: Jin, S., InTech Publisher, ISBN 978-953-51-1144-3, 354 pp., doi:10.5772/3439, 2013.

Kursinski, E. R., Hajj, G. A., Schofield, J. T., Kursinski, E. R., Hajj, G. A., Schofield, J. T., Linfield, R. P., and Hardy, K. R.: Observing Earth's atmosphere with radio occultation measurements using the global positioning system, J. Geophys. Res., 102, 2342923465, 1997.

Lindal, G. F., Wood, G. E., Hotz, H. B., Sweetnam, D. N., Eshleman, V. R., and Tyler, G. L.: The atmosphere of Titan: an analysis of the Voyager 1 radio occultation measurements, Icarus, 53, 348-363, 1983.

Lindal, G. F., Lyons, J. R., Sweetnam, D. N., Eshleman, V. R., Hinson, D. P., and Tyler, G. L.: The atmosphere of Uranus: results of radio occultation measurements with Voyager, J. Geophys. Res., 92, 14987-15001, 1987.

Liou, Y. A. and Pavelyev, A. G.: Simultaneous observations of radio wave phase and intensity variations for locating the plasma layers in the ionosphere, Geophys. Res. Lett., 33, L23102, doi:10.1029/2006GL027112, 2006.

Liou, Y.-A., Pavelyev, A. G., Huang, C.-Y., Igarashi, K., and Hocke, K.: Simultaneous observation of the vertical gradients of refractivity in the atmosphere and electron density in the lower ionosphere by radio occultation amplitude method, Geophys. Res. Lett., 29, 43-1-43-4, doi:10.1029/2002GL015155, 2002.

Liou, Y.-A., Pavelyev, A. G., Huang, C.-Y., Igarashi, K., Hocke, K., and Yan, S. K.: Analytic method for observation of the GW using RO data, Geophys. Res. Lett., 30, ASC 1-1-1-5, doi:10.1029/2003GL017818, 2003.

Liou, Y. A., Pavelyev, A. G., Pavelyev, A. A., Wickert, J., and Schmidt, T.: Analysis of atmospheric and ionospheric structures using the GPS/MET and CHAMP radio occultation data base: a methodological review, GPS Solut., 9, 122-143, 2005a.

Liou, Y. A., Pavelyev, A. G., and Wickert, J.: Observation of the gravity waves from GPS/MET radio occultation data, J. Atmos. Sol.-Terr. Phys., 67, 219-228, doi:10.1016/j.jastp.2004.08.001, $2005 b$.

Liou, Y. A., Pavelyev, A. G., Liu, S.-F., Pavelyev, A. A., Yen, N., Huang, C.-Y., and Fong, C.-J.: FORMOSAT-3/COSMIC GPS radio occultation mission: preliminary results, IEEE T. Geosci. Remote, 45, 3813-3826, 2007.

Liou, Y. A., Pavelyev, A. G., Matyugov, S. S., Yakovlev, O. I., and Wickert, J.: Radio Occultation Method for Remote Sensing of the Atmosphere and Ionosphere, edited by: Liou, Y. A., IN-TECH, In-The Olajnica 19/2, 32000 Vukovar, Croatia, 170, 45 pp., ISBN 978-953-7619-60-2, 2010.

Manzini, E. and Bengtsson, L.: An observing system simulation experiment for climate monitoring with GNSS radio occultation data: setup and test bed study, J. Geophys. Res., 113, D11108, doi:10.1029/2007JD009231, 2008.

Marouf, E. A. and Tyler, G. L.: Microwave edge diffraction by features in Saturn's rings: observations with Voyager 1, Science, 217, 243-245, 1982.

Melbourne, W. G.: Radio Occultations Using Earth Satellites: A Wave Theory Treatment, Jet Propulsion Laboratory California Institute of Technology, Monograph 6, Deep Space Communications and Navigation Series, edited by: Yuen, J. H., the Deep Space Communications and Navigation Systems Center of Excellence Jet Propulsion Laboratory California Institute of Technology, 610 pp., 2004.

Melbourne, W. G., Davis, E. S., Duncan, C. B., Hajj, G. A., Hardy, K. R., Kursinski, E. R., Meehan, T. K., Young, L. E., and Yunck, T. P.: The application of spaceborne GPS to atmospheric limb sounding and global change monitoring, JPL Publication, 94-18, 147 pp., 1994.

Mortensen, M. D. and Høeg, P.: Inversion of GPS occultation measurements using Fresnel diffraction theory, Geophys. Res. Lett., 25, 2441-2444, 1998.

Mortensen, M. D., Lifield, R. P., and Kursinski, E. R.: Vertical resolution approaching $100 \mathrm{~m}$ for GPS occultations of the Earth's atmosphere, Radio Sci., 36, 1475-1484, 1999.

Pavelyev, A. G.: On possibility of radio holographic investigation on communication link satellite-to-satellite, J. Commun. Technol. Electron., 43, 126-132, 1998.

Pavelyev A. G.: The Principle of the Locality and Radio Occultation Method for Remote Sensing of Layers in the Atmosphere 
and Ionosphere on Earth and Other Planets, Doklady Physics, 58, 375-378, 2013.

Pavelyev, A. G. and Kucherjavenkov, A. I.: Refractive attenuation in the planetary atmospheres, Radio Eng. Electron. P., 23, 13-19, 1978.

Pavelyev, A. G., Volkov, A. V., Zakharov, A. I., Krytikh, S. A., and Kucherjavenkov A. I.: Bistatic radar as a tool for Earth observation using small satellites, Acta Astronautica, 39, 721-730, 1996.

Pavelyev, A. G., Liou, Y.-A., Huang, C. Y., Reigber, C., Wickert, J., Igarashi, K., and Hocke, K.: Radio holographic method for the study of the ionosphere, atmosphere and terrestrial surface using GPS occultation signals, GPS Solut., 6, 101-108, 2002.

Pavelyev, A. G., Liou, Y. A., and Wickert, J.: Diffractive vector and scalar integrals for bistatic radio-holographic remote sensing, Radio Sci., 39, RS4011, doi:10.1029/2003RS002935, 2004.

Pavelyev, A. G., Liou, Y. A., Wickert, J., Schmidt, T., Pavelyev, A. A., and Liu, S. F.: Effects of the ionosphere and solar activity on radio occultation signals: application to CHAllenging Minisatellite Payload satellite observations, J. Geophys. Res., 112, A06326, doi:10.1029/2006JA011625, 2007.

Pavelyev, A. G., Wickert, J., and Liou, Y.-A.: Localization of plasma layers in the ionosphere based on observing variations in the amplitude and phase of radiowaves along the satellite-to-satellite path, Radiophys. Quantum El., 51, 1-8, 2008a.

Pavelyev, A. G., Liou, Y.-A., Wickert, J., Pavelyev, A. A., Schmidt, T., Igarashi, K., and Matyugov, S. S.: Location of layered structures in the ionosphere and atmosphere by use of GPS occultation data, Adv. Space Res., 42, 224-228, 2008b.

Pavelyev, A. G., Liou, Y. A., Wickert, J., Gavrik, A. L., and Lee, C. C.: Eikonal acceleration technique for studying of the Earth and planetary atmospheres by radio occultation method, Geophys. Res. Lett., 36, L21807, doi:10.1029/2009GL040979, 2009.

Pavelyev, A. G., Liou, Y.-A., Wickert, J., Schmidt, T., Pavelyev, A. A., and Matyugov, S. S.: Phase acceleration: a new important parameter in GPS occultation technology, GPS Solut., 14, 3-14, doi:10.1007/s10291-009-0128-1, 2010a.

Pavelyev, A. G., Liou, Y. A., Wickert, J., Zhang, K., Wang, C.-S., and Kuleshov, Y.: Analytical model of electromagnetic waves propagation and location of inclined plasma layers using occultation data, Prog. Electromagn. Res., 106, 177-202, doi:10.2528/PIER10042707, 2010b.

Pavelyev, A. G., Zhang, K., Matyugov, S. S., Liou, Y. A., Wang, C. S., Yakovlev, O. I., Kucherjavenkov, I. A., and Kuleshov, Y.: Analytical model of bistatic reflections and radio occultation signals, Radio Sci., 46, RS1009, doi:10.1029/2010RS004434, 2011.

Pavelyev, A. G., Liou, Y. A., Zhang, K., Wang, C. S., Wickert, J., Schmidt, T., Gubenko, V. N., Pavelyev, A. A., and Kuleshov, Y.: Identification and localization of layers in the ionosphere using the eikonal and amplitude of radio occultation signals, Atmos. Meas. Tech., 5, 1-16, doi:10.5194/amt-5-1-2012, 2012.

Pavelyev, A. G., Zhang, K., Liou, Y. A., Pavelyev, A. A., Wang, C.S., Wickert, J., Schmidt, T., and Kuleshov Y.: Principle of Locality and Analysis of Radio Occultation Data, IEEE T. Geosci. Remote, 51, 3240-3249, doi:10.1109/TGRS.2012.2225629, 2013.

Rius, A., Ruffini, G., and Romeo, A.: Analysis of ionospheric electron density distribution from GPS/Met occultations, IEEE T. Geosci. Remote, 36, 383-394, 1998.

Schmidt, T., Heise, S., Wickert, J., Beyerle, G., and Reigber, C.: GPS radio occultation with CHAMP and SAC-C: global moni- toring of thermal tropopause parameters, Atmos. Chem. Phys., 5, 1473-1488, doi:10.5194/acp-5-1473-2005, 2005.

Sokolovskiy, S. V.: Inversion of RO amplitude data, Radio Sci., 35, 97-105, 2000.

Sokolovskiy, S. V., Schreiner, W., Rocken, C., and Hunt, D.: Detection of high-altitude ionospheric irregularities with GPS/MET, Geophys. Res. Lett., 29, 621-625, 2002.

Steiner, A. K. and Kirchengast, G.: GW spectra from GPS/MET occultation observations, J. Atmos. Ocean. Tech., 17, 495-503, 2000.

Steiner, A. K., Kirchengast, G., and Ladreiter, H. P.: Inversion, error analysis, and validation of GPS/MET occultation data, Ann. Geophys., 17, 122-138, doi:10.1007/s00585-999-0122-5, 1999.

Steiner, A. K., Kirchengast, G., Foelsche, U., Kornblueh, L., Manzini, E., and Bengtsson, L.: GNSS occultation sounding for climate monitoring, Phys. Chem. Earth Pt. A, 26, 113-124, 2001.

Syndergaard, S.: Modeling the impact of the Earth's oblateness on the retrieval of temperature and pressure profiles from limb sounding, J. Atmos. Sol.-Terr. Phy., 60, 171-180, 1998.

Syndergaard, S.: Retrieval analysis and methodologies in atmospheric limb sounding using the GNSS radio occultation technique, DMI Sci. Rep. 99-6, Danish Met. Inst., Copenhagen, Denmark, 131 pp., available at: http://www.cosmic.ucar.edu/ groupAct/references/Sr99-6.pdf (last access: 10 January 2015), 1999.

Von Engeln, A., Andresa, Y., Marquardt, C., and Sancho, F.: GRAS Radio Occultation on-board of Metop, Adv. Space Res., 47, 336347, doi:10.1016/j.asr.2010.07.028, 2011.

Vorob'ev, V. V. and Krasilnikova, T. G.: Estimation of accuracy of the atmosphere refractive index recovery from Doppler shift measurements at frequencies used in the NAVSTAR system, Izv. Russ. Acad. Sci., 29, 602-609, 1994 (Engl. Transl.).

Vorob'ev, V. V., Gurvich, A. S., Kan, V., Sokolovskiy, S. V., Fedorova, O. V., and Shmakov, A. V.: The structure of the ionosphere from theGPS-"Microlab-1" radio occultation data: preliminary results, Cosmic Res., 4, 74-83, 1997 (in Russian).

Ware, R., Exner, M., Feng, D., Gorbunov, M., Hardy, K., Herman, B., Kuo, Y.-H., Meehan, T., Melbourne, W., Rocken, C., Schreiner, W., Sokolovskiy, S., Solheim, F., Zou, X., Anthes, R., Businger, S., and Trenberth, K.: GPS soundings of the atmosphere from low earth orbit: preliminary results, B. Am. Meteorol. Soc., 77, 19-40, 1996.

Wickert, J., Pavelyev, A. G., Liou, Y. A., Schmidt, T., Reigber, C., Igarashi, K., Pavelyev, A. A., and Matyugov, S.: Amplitude scintillations in GPS signals as a possible indicator of ionospheric structures, Geophys. Res. Lett., 31, L24801, doi:10.1029/2004GL020607, 2004.

Wickert, J., Reigber, C., Beyerle, G., Konig, R., Marquardt, C., Schmidt, T., Grunwaldt, L., Galas, R., Meehan, T. K., Melbourne, W. G., and Hocke, K.: Atmosphere sounding by GPS radio occultation: first results from CHAMP, Geophys. Res. Lett., 28, 3263-3266, 2001.

Wickert, J., Beyerle, G., König, R., Heise, S., Grunwaldt, L., Michalak, G., Reigber, Ch., and Schmidt, T.: GPS radio occultation with CHAMP and GRACE: A first look at a new and promising satellite configuration for global atmospheric sounding, Ann. Geophys., 23, 653-658, doi:10.5194/angeo-23-653-2005, 2005.

Wickert, J., Schmidt, T., Michalak, G., Heise, S., Arras, C., Beyerle, G., Falck, C., König, R., Pingel, D., and Rothacher, M.: GPS ra- 
dio occultation with CHAMP, GRACE-A, SAC-C, TerraSAR-X, and FORMOSAT-3/COSMIC: brief review of results from GFZ, in: New Horizons in Occultation Research: Studies in Atmosphere and Climate, edited by: Steiner, A., Pirscher, B., Foelsche, U., and Kirchengast, G., Springer, 3-16, 2009.

Yakovlev, O. I.: Space Radio Science, Taylor and Francis, London, 306 pp., 2002.

Yakovlev, O. I., Pavelyev, A. G., and Matyugov, S. S.: Radio Occultation Monitoring of the Atmo-sphere and Ionosphere, URSS Edition, Moscow, 206 pp., ISBN 978-5-397-01227-0, 2010 (in Russian).

Yunck, T. P., Lindal, G. F., and Liu, C.-H.: The role of GPS in precise Earth observation, in: Proc. IEEE Position Location and Navigation Symposium (PLANS 88), 29 November-December, 1988.
Yunck, T. P., Liu, C.-H., and Ware, R.: A history of GPS sounding, Terr. Atmos. Ocean. Sci., 11, 1-20, 2000.

Zhang, K., Zhang, S., Le Marshall, J., Kirchengast, G., Norman, R., Ying Li, Liu, C., and Carter, B.: A new Australian GNSS radio occultation data processing platform, in: IGNSS 2013 Symposium Proc., Gold Coast, Australia, ISBN 978-0-646-90640-9, Pap.85/12p., 2013.

Zus, F., Grunwaldt, L., Heise, S., Michalak, G., Schmidt, T., and Wickert, J.: Atmosphere sounding by GPS radio occultation: first results from TanDEM-X and comparison with TerraSARX, Adv. Space Res., 53, 272-279, doi:10.1016/j.asr.2013.11.013, 2014. 